\title{
Endothelial cell CD36 optimizes tissue fatty acid uptake
}

\author{
Ni-Huiping Son, ${ }^{1}$ Debapriya Basu, ${ }^{1}$ Dmitri Samovski, ${ }^{2}$ Terri A. Pietka, ${ }^{2}$ Vivek S. Peche, ${ }^{2}$ Florian Willecke, ${ }^{1}$ Xiang Fang, ${ }^{1}$ \\ Shui-Qing Yu, ${ }^{1}$ Diego Scerbo, ${ }^{1}$ Hye Rim Chang, ${ }^{1}$ Fei Sun, ${ }^{1}$ Svetlana Bagdasarov, ${ }^{1}$ Konstantinos Drosatos, ${ }^{3}$ Steve T. Yeh, ${ }^{4}$ \\ Adam E. Mullick, ${ }^{4}$ Kooresh I. Shoghi, ${ }^{5}$ Namrata Gumaste, ${ }^{1}$ KyeongJin Kim, ${ }^{6}$ Lesley-Ann Huggins, ${ }^{1}$ Tenzin Lhakhang, ${ }^{7}$ \\ Nada A. Abumrad, ${ }^{2}$ and Ira J. Goldberg ${ }^{1}$
}

'Division of Endocrinology, Diabetes and Metabolism, New York University School of Medicine, New York, New York, USA. ²Department of Medicine, Washington University School of Medicine, St. Louis, Missouri, USA. ${ }^{3}$ Department of Pharmacology, Lewis Katz School of Medicine at Temple University, Philadelphia, Pennsylvania, USA. ${ }^{4}$ lonis Pharmaceuticals Inc., Carlsbad, California, USA. ${ }^{5}$ Department of Radiology, Washington University School of Medicine, St. Louis, Missouri, USA. ${ }^{6}$ Division of Endocrinology, Columbia University Medical Center, New York, New York, USA. ${ }^{7}$ NYU Genome Technology Center, NYU Langone Medical Center, New York, New York, USA.

\begin{abstract}
Movement of circulating fatty acids (FAs) to parenchymal cells requires their transfer across the endothelial cell (EC) barrier. The multiligand receptor cluster of differentiation 36 (CD36) facilitates tissue FA uptake and is expressed in ECs and parenchymal cells such as myocytes and adipocytes. Whether tissue uptake of FAs is dependent on EC or parenchymal cell CD36, or both, is unknown. Using a cell-specific deletion approach, we show that EC, but not parenchymal cell, CD36 deletion increased fasting plasma FAs and postprandial triglycerides. EC-Cd36-KO mice had reduced uptake of radiolabeled longchain FAs into heart, skeletal muscle, and brown adipose tissue; these uptake studies were replicated using ["1'C]palmitate PET scans. High-fat diet-fed EC-CD36-deficient mice had improved glucose tolerance and insulin sensitivity. Both EC and cardiomyocyte (CM) deletion of CD36 reduced heart lipid droplet accumulation after fasting, but CM deletion did not affect heart glucose or FA uptake. Expression in the heart of several genes modulating glucose metabolism and insulin action increased with EC-CD36 deletion but decreased with CM deletion. In conclusion, EC CD36 acts as a gatekeeper for parenchymal cell FA uptake, with important downstream effects on glucose utilization and insulin action.
\end{abstract}

\section{Introduction}

Long-chain fatty acids (LCFAs) provide cellular energy and are precursors for membrane components and intracellular lipid stores. Their bioactive derivatives influence gene expression and signal transduction pathways. Distribution of LCFAs among organs, especially muscle, adipose tissue, and liver, is key to maintaining metabolic homeostasis (1). In the bloodstream, LCFAs circulate as unesterified free fatty acids (FFAs) bound to albumin or as triglyceride (TG) and phospholipid components of lipoproteins. Tissue uptake of LCFAs requires transfer across an endothelial cell (EC) barrier, but the molecular events necessary for this remain unknown.

In cultured parenchymal cells, LCFA uptake occurs via a saturable receptor-mediated pathway and a nonsaturable pathway thought to reflect passive diffusion $(2,3)$. Several proteins have been implicated in facilitating LCFA accumulation, including enzymes involved in FA acylation and the scavenger receptor cluster of differentiation 36 (CD36) (4). CD36 (also called SR-B2) is a multifunctional protein involved in apoptotic cell uptake, signal transduction, cellular adhesion, angiogenesis, and immune function (5). It was first linked to lipid metabolism when it was shown to function as a macrophage receptor for oxidized LDL (6) and as an adipocyte receptor/transporter for LCFAs (7). LCFA binding to the CD36 ectodomain is followed by FA transfer to the plasma

Conflict of interest: The authors have declared that no conflict of interest exists. Submitted: December 18, 2017; Accepted: July 18, 2018.

Reference information: J Clin Invest. 2018;128(10):4329-4342.

https://doi.org/10.1172/JCI99315. membrane through an internal tunnel that runs the length of the protein, as shown by the crystal structure of the CD36-LCFA complex (8). CD36 also functions as a LCFA sensor, influencing activation of PPARs (9) and AMPK (10).

In mice, germline $\mathrm{CD} 36$ deficiency reduces acute accumulation of LCFAs in the heart, skeletal muscle, and adipose tissues (11). Moreover, $C d 36^{-/}$mice have increased fasting levels of FFAs $(11,12)$ and delayed clearance of postprandial lipids (11-13). The defect in LCFA uptake is also observed in CD36-deficient humans (14). Furthermore, a common haplotype at the CD36 locus is associated with greater circulating FFA levels (15). Whether the defect in FA uptake is consequent to reduced CD36 expression in ECs, parenchymal cells, or both is unknown. We bred mice with $\mathrm{CD} 36$ deletion specific to ECs (EC-Cd36--) or cardiomyocytes (CM-Cd36--) to determine which cell type(s) regulate organ/ tissue accumulation of LCFAs and TGs. Our data show that EC CD36 is needed for optimal movement of LCFAs from the circulation to cardiomyocytes (CMs). LCFA uptake was also diminished in brown adipose tissue (BAT) and skeletal muscle, but not liver, of EC-Cd36- mice. Loss of EC CD36 improved glucose tolerance and insulin sensitivity and increased glucose uptake during fasting in heart, skeletal muscle, and white adipose tissue (WAT). The data reveal that CD36-mediated FA transport across ECs limits tissue FA uptake and that its loss leads to metabolic effects in parenchymal cells.

\section{Results}

CD36 is abundantly expressed in vascular ECs and CMs in human and mouse hearts. We investigated CD36 distribution in human and mouse heart tissues and assessed the fidelity of our cell-spe- 
A

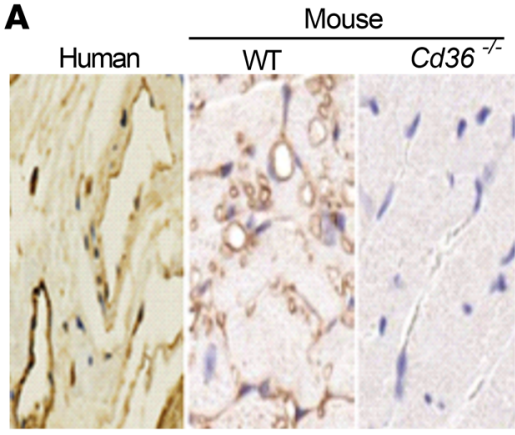

B

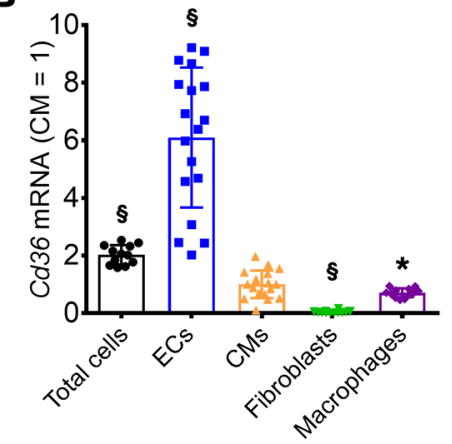

C

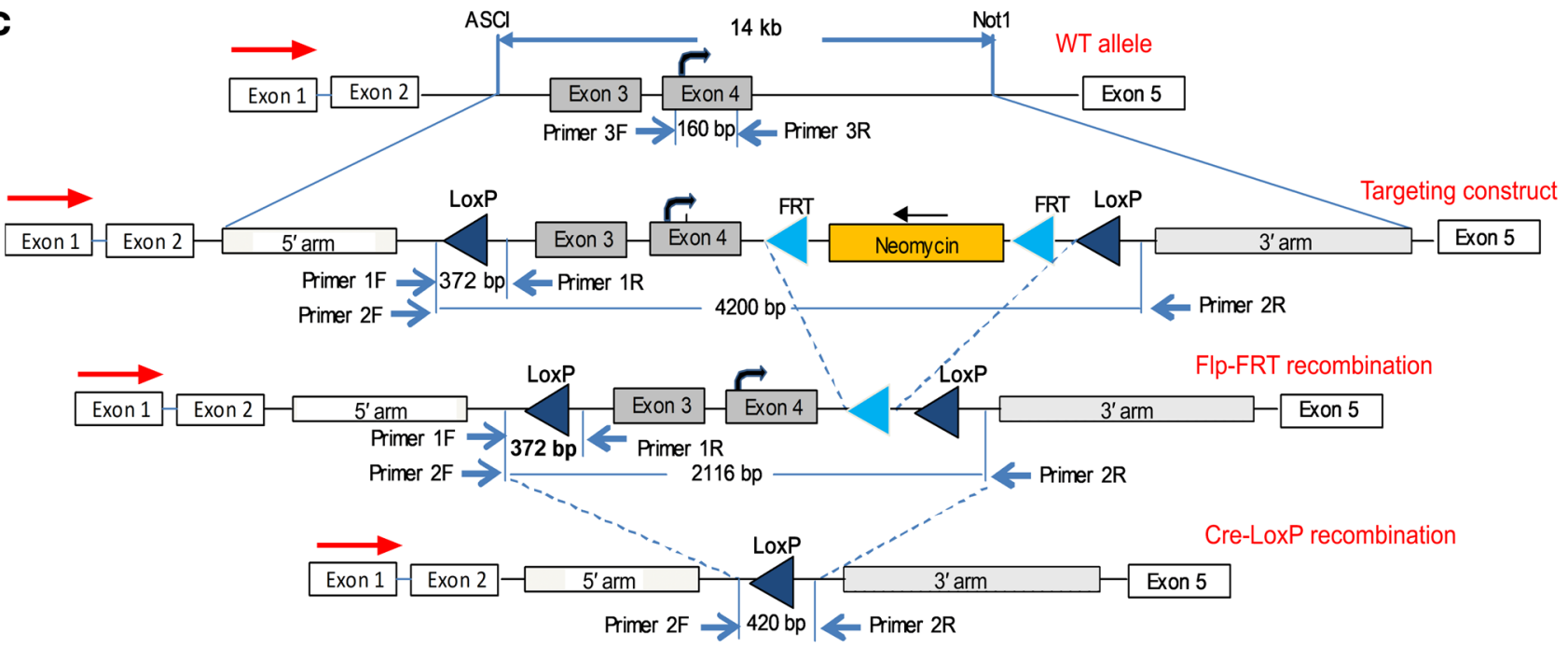

D

F WT allele
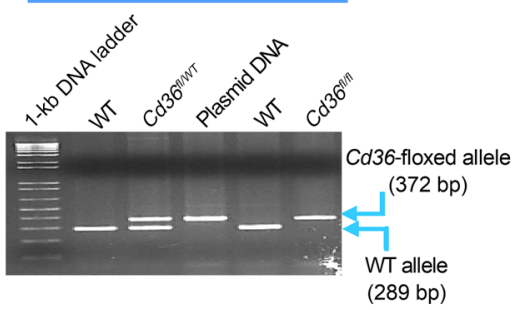

E Heart tissue DNA

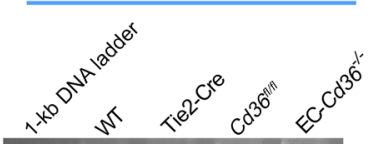

$\stackrel{2}{=} \longrightarrow$

<Cd36-null allele
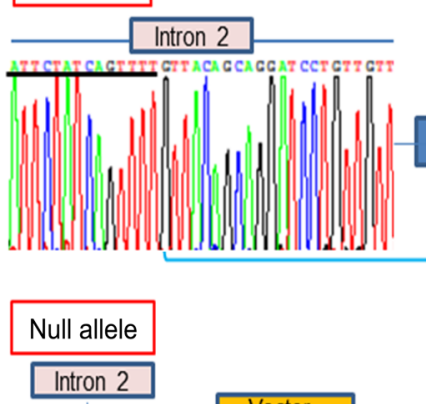

Exon 3

Intron 3

Exon 4

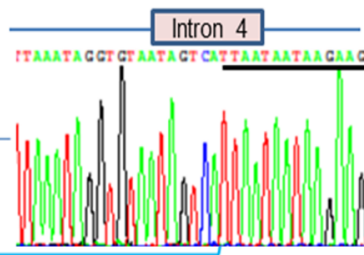

Cre-LoxP recombination

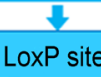

ntron 4

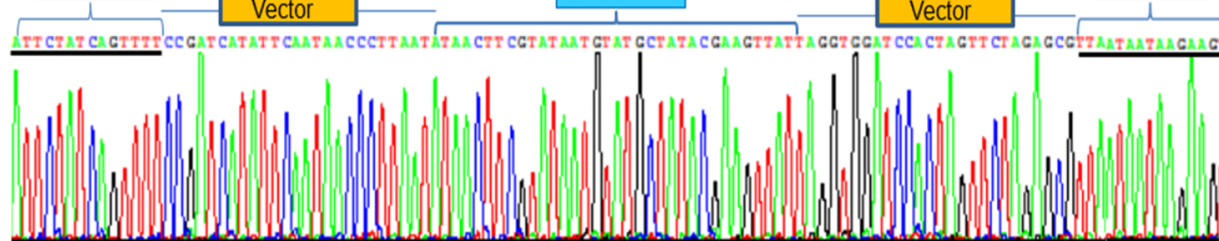

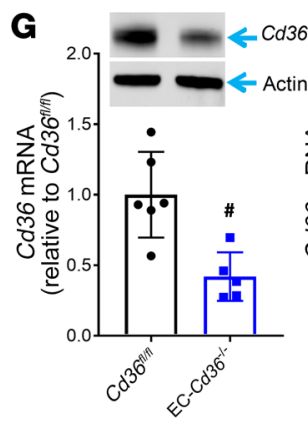
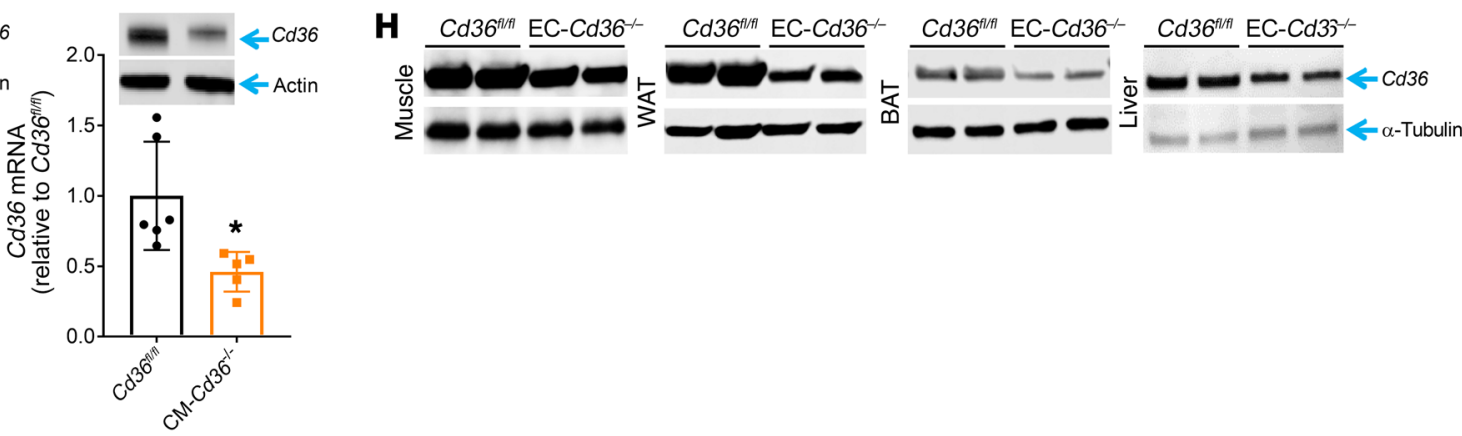
Figure 1. CD36 distribution in human and mouse hearts and creation of EC- and CM-Cd36 $6^{-/-}$mice. (A) CD36 antibody staining of CMs and blood vessel ECs from human and mouse hearts. Original magnification, $\times 20$. (B) Male mouse heart suborgan fractionation showing Cd36 mRNA levels in different cell types $(n=12-18)$. Data represent mean ratios normalized to $\mathrm{CM}-\mathrm{Cd} 3 \mathrm{6}^{-/-}$(set at 1.0). (C) Cenomic structure of murine $\mathrm{Cd} 36$, the targeting vector, and the mutated allele. Gray boxes represent the numbered murine Cd36 exons. Red arrows indicate transcription orientation. Black bent arrows indicate the translation start site. PCR primers (blue arrows) were chosen to differentiate between the WT genomic allele and the homologously recombined allele. (D) PCR of tail genomic DNA (using primer 1) and (E) heart tissue DNA (using primer 2) from $C d 36^{f / / f l}, \mathrm{EC}-\mathrm{Cd} 36^{-1-}$ and $\mathrm{CM}-\mathrm{Cd} 36^{-/-}$mice. (F) PCR product sequencing showing ablation of exons 3 and 4 of $C d 36$ gene after Cre-mediated recombination. (C) Heart Cd36 mRNA in EC-Cd36-/- and CM-Cd36-/- mice quantified by qRT-PCR using primer 3 ( $n=5-6$, mean \pm SD). Data are corrected for 185 rRNA and normalized to $\mathrm{Cd} 36^{f / f f}$ controls. Immunoblots show $\mathrm{CD} 36$ in $\mathrm{EC}-\mathrm{Cd} 36^{-/-}$and $\mathrm{CM}-\mathrm{Cd} 36^{-/-}$mouse hearts. $(\mathrm{H})$ Immunoblots of $\mathrm{CD} 36$ in $\mathrm{EC}-\mathrm{Cd} 36^{-/-}$muscle, WAT, BAT, and liver. ${ }^{*} P<0.05,{ }^{\#} P<0.01$ and ${ }^{\S} P<0.001$ compared with Cd $36^{f / f l}$ controls; 1-way ANOVA with Dunnett's multiple comparisons test.

cific deletions. We found that CD36 was present in ECs and CMs in both human and mouse hearts (Figure 1A) and that staining was most intense in small blood vessels, weaker in larger arteries, and absent in total $\mathrm{Cd} 36^{-/-}$mouse hearts. In WT mouse hearts subjected to Langendorff perfusion and subsequent suborgan fractionation using gradient density separation and FACS, we found that $C d 36$ mRNA levels were significantly higher in ECs than in CMs from both male (Figure 1B) and female mice (Supplemental Figure 1A; supplemental material available online with this article; https:// doi.org/10.1172/JCI99315DS1). ISH data showed Cd36 mRNA expression in WT, but not total $C d 36^{-/}$, mouse hearts, with the most intense signals detected in capillary ECs (Supplemental Figure 1B).

$C D 36 \mathrm{mRNA}$ and protein are specifically reduced in EC- and $\mathrm{CM}-\mathrm{Cd} 36^{-/-}$mouse hearts. To determine the contribution of EC versus CM CD36 to heart FFA uptake, we created a $C d 36^{f l / f l}$ mouse line (Figure 1C). We crossed $C d 36^{f / f l}$ mice with Tie2-Cre mice to generate $\mathrm{EC}-\mathrm{Cd} 36^{-/}$mice and with $\mathrm{MHC}$-Cre mice to generate $\mathrm{CM}-\mathrm{Cd} 36^{-/-}$mice. Both groups were fertile and appeared normal. We used PCR to detect $C d 36^{f / f l}$ and $C d 36^{-/-}$alleles from tail (Figure 1D) and heart (Figure 1E) DNA. PCR product sequencing from Cd36-null fragments showed ablation of exons 3 and 4 of the $C d 36$ gene after Cre-mediated recombination (Figure 1F). Cd36 mRNA and protein levels were reduced by more than $50 \%$ in the hearts of EC-Cd36/- mice compared with levels in $C d 36^{f / f l}$ mouse hearts; $\mathrm{CM}-\mathrm{Cd} 36^{-/}$mice also had an approximately $50 \%$ reduction of Cd36 mRNA in the heart, with parallel reductions in protein content (Figure 1G). The approximately $50 \%$ reduction with either the $\mathrm{EC}$ or $\mathrm{CM} \mathrm{KO}$ suggests that total heart CD36 is equally divided in these 2 cell types. EC-Cd36 $6^{--}$mice had reduced $C d 36$ mRNA in skeletal muscle, BAT, and liver (Supplemental Figure 1, C-E), and we measured parallel reductions in CD36 protein in skeletal muscle, adipose tissue (WAT and BAT), and liver (Figure 1H). Immunohistologic analysis of cardiac ventricular sections stained with polyclonal rabbit CD36 antibody confirmed CD36 ablation specificity to capillary ECs from EC-Cd36/- mice (Figure 2A). These mice had significantly reduced $C d 36$ levels in the lungs, which are particularly rich in ECs (Supplemental Figure 1F). To verify that CD36 is specifically deleted in ECs, we double stained EC-Cd36-/- heart tissues with CD36 and the EC marker CD31. Both CD36 and CD31 colocalized to capillary ECs of $C d 36^{f / f l}$ control mice, while CD36 protein was undetected in CD31-positive EC-Cd36 ${ }^{-/-}$heart tissues (Figure 2B). We further confirmed the specificity of the EC versus CM deletions by immunofluorescence of isolated ECs and $\mathrm{CMs}$ from lung and heart tissues. We found that CMs from $\mathrm{EC}-\mathrm{Cd} 36^{-/}$hearts contained normal levels of CD36 protein, whereas CD36 was undetectable in CM-Cd36 $6^{-/-}$heart CMs (Figure 2C). Primary ECs isolated from the lungs of $C d 36^{f / f l}$ and inducible VE-cadherin $\mathrm{Cd}_{36^{-/}}$mice (16) were stained for both VE-cadherin, an endothelium-specific molecule, and CD36 and showed an absence of CD36 staining in VE-cadherin-positive ECs from EC-Cd36 $6^{-/}$mice (Figure 2D).

To verify that $C d 36$ EC deletion did not cause off-target effects, we confirmed the presence of CD36 in CMs isolated from EC-Cd36/mice and analyzed the cells by Western blotting and quantitative real-time PCR (qRT-PCR) (Supplemental Figure 1G). These data proved that CD36 was deleted in ECs but not parenchymal cells.

To determine whether CD36 deletion modified the metabolic gene profile in ECs, we isolated ECs from $\mathrm{Cd} 36^{f / f l}, \mathrm{EC}-\mathrm{Cd} 36^{-/}$, and $\mathrm{CM}-\mathrm{C} d 36^{-/}$mouse hearts using FACS. Genes involved in glucose metabolism were not altered significantly in ECs from the 3 groups (Supplemental Figure 1H). We also found no changes in FA oxidation genes in ECs with CD36 deletion, consistent with glucose being the primary substrate for ATP production in these cells. Thus, CD36 is not a major regulator of lipid or glucose metabolism in ECs.

We used laser capture microdissection (LCM) technology to isolate CMs and determine the expression of glucose and FA metabolism genes. Glut1 mRNA increased, while pyruvate dehydrogenase kinase $4(P d k 4)$ and carnitine palmitoyl transferase 1 (Cpt1) were decreased in the EC-Cd36/- CMs (Supplemental Figure 1I). These changes suggest a switch from FA to glucose use.

CD36 deletion in ECs increases plasma FFA and postprandial TG levels, while decreasing plasma glucose levels and enhancing insulin sensitivity. Deletion of EC Cd36 increased fasting plasma FFAs (Figure $3 \mathrm{~A}$ ), a phenotype similar to that of mice with global CD36 deletion (12). Fasting plasma total cholesterol (TC), TGs, glycerol, and lipoprotein lipids (Supplemental Figure 2, A-E) were unchanged in 4-month-old male EC-Cd36/- mice compared with levels in $C d 36^{f / f l}$ controls. However, age-matched female EC-Cd36 $6^{-/-}$mice showed decreased plasma glucose together with increased fasting plasma TC, FFA, and TGs associated with an increase in VLDL-TG and HDL-TC (Supplemental Figure 3, A-F). These sex differences in lipid levels might reflect previously reported sex differences in the regulation of tissue CD36 expression and muscle FA metabolism $(17,18)$. We found that loss of CD36 in CMs did not alter circulating levels of FFAs or glucose (Figure 3, A and B). Therefore, loss of CD36 only in ECs reproduced many of the overall metabolic effects seen with total-body CD36 KO.

Postprandial TG levels in response to fat intake were higher in EC-Cd36 $6^{-/}$mice. Plasma TG levels 2, 4, and 6 hours after olive oil gavage were higher in $\mathrm{EC}-\mathrm{Cd} 36^{-/-}$mice than in $\mathrm{Cd} 36^{f / / f l}$ mice (Figure $3 \mathrm{C}$ ). We detected a greater than 2-fold increase in the AUC for TG clearance $\left(\mathrm{AUC}_{\mathrm{TG}}\right.$ ) in EC-Cd36/- mice (Figure $3 \mathrm{C}$ ), suggesting that EC CD36 is involved in the rapid clearance of circulating TGs. In contrast, loss of CM CD36 did not alter TG excursion (Figure $3 \mathrm{C})$. Slower postprandial TG clearance is characteristic of total 
A

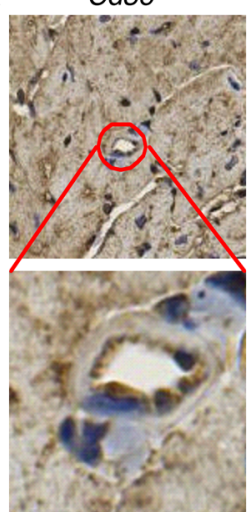

C

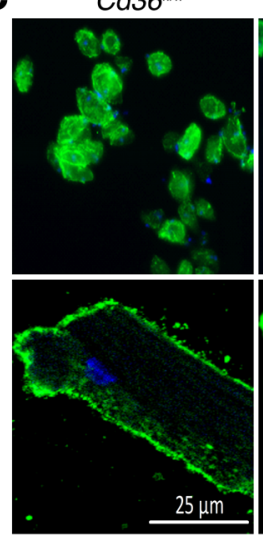

$\mathrm{EC}-\mathrm{Cd} 36^{\prime \prime}$

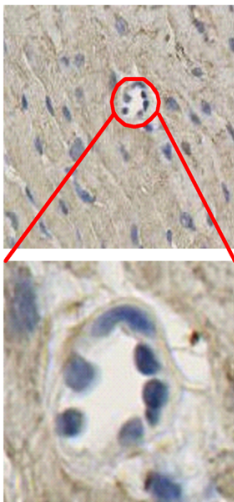

$\mathrm{EC}-\mathrm{Cd} 36^{--}$

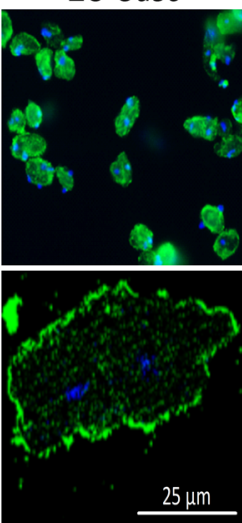

$\mathrm{CM}-\mathrm{Cd} 36^{-1}$

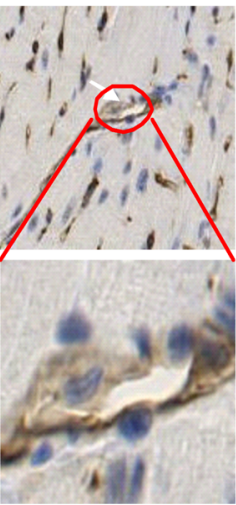

$\mathrm{CM}-\mathrm{Cd} 36^{-1-}$

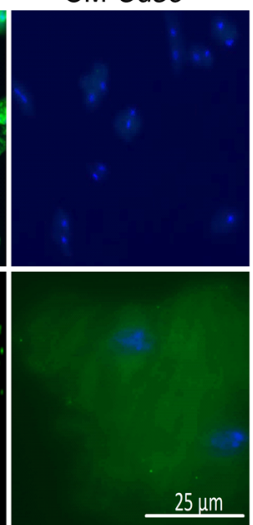

B
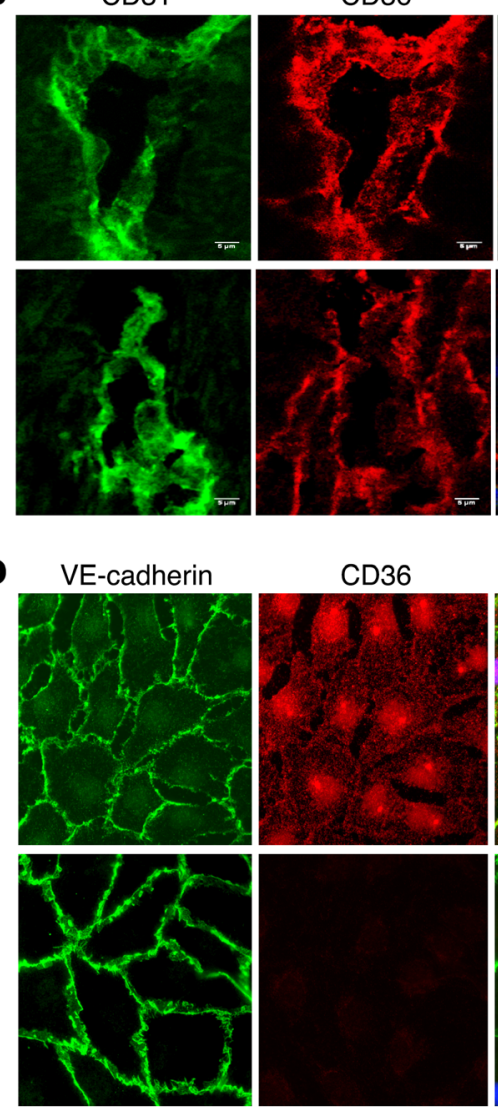

CD36

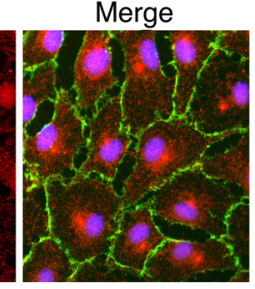

$\operatorname{cd} 36^{i t /+1}$

Merge

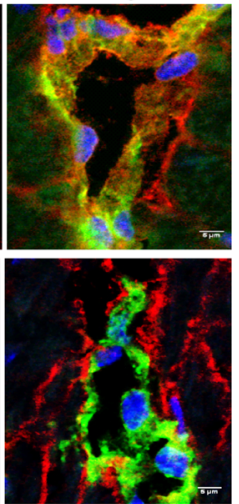

Cd36

$\mathrm{EC}-\mathrm{Cd} 36^{-1-}$
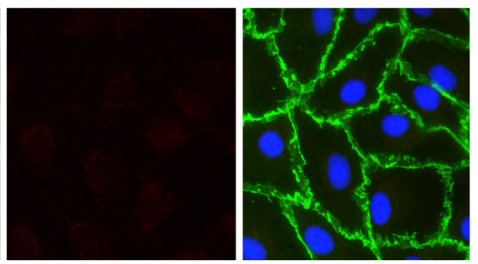

$\mathrm{EC}-\mathrm{Cd} 36^{-/-}$

Figure 2. Immunocytochemistry and immunofluorescence staining of heart tissue and isolated ECs and CMs. (A) CD36 staining of heart tissue from Cd $36^{f / / f l}, \mathrm{EC}-\mathrm{Cd} 36^{-/-}$, and CM-Cd36 $6^{-/-}$mice (top) and higher-magnification images of CD36 staining of vascular endothelium and CMs (bottom). Original magnification, $\times 40$ (top) and $\times 400$ (bottom). (B) Cd36 $6^{f / f l}$ and EC-Cd36-/- heart sections were double stained with anti-CD31 (green) and anti-CD36 (red) antibodies. Scale bars: $5 \mu \mathrm{m}$. (C) Isolated CMs from Cd36 $6^{f l / f l}, \mathrm{EC}-\mathrm{Cd} 36^{-/-}$, and CM-Cd36-/- mouse hearts stained with anti-CD36 antibody. Scale bars: $25 \mu \mathrm{m}$. (D) Isolated ECs from $C d 36^{f / / f l}$ and EC-Cd36-/- mice stained with anti-VE-cadherin (green) and anti-CD36 (red) antibodies. Original magnification, $\times 60$.

CD36 deficiency in mice (13) and humans (19) and may reflect greater product inhibition of lipoprotein lipase (LpL) by FFAs.

Glucose levels and insulin sensitivity are altered by loss of CD36 in ECs. EC-Cd36-- mice had decreased 16-hour fasting glucose levels and an improved oral glucose tolerance test (OGTT) (Figure 3D), as was previously found in total $C d 36 \mathrm{KOs}$ (20). The AUC for OGTT ( $\mathrm{AUC}_{\mathrm{OGTT}}$ ) for EC-Cd36-- mice was $24 \%$ lower than for $C d 36^{A / f l}$ mice (Figure 3D). Insulin tolerance tests (ITTs) in 2-hourfasted mice showed that the more rapid glucose removal was due to greater insulin sensitivity (Figure 3E).

We generated a second endothelial KO line by crossing $C d 36^{A / f / 1}$ mice with VE-cadherin-Cre (VE-Cd36--) mice. In this model, plasma glucose levels were decreased, while FFAs were increased, similar to the effects seen in Tie2-Cre EC-Cd36-/ mice (Supplemental Figure $4, \mathrm{~A}$ and $\mathrm{B}$ ).

To confirm that the changes in FFA and glucose levels were not due to off-target CD36 deletion, we created myeloid CD36-KO mice using the LysM-Cre transgene. The deletion did not reduce glucose or increase circulating FFA levels (Supplemental Figure 5, A and B).

CD36 deletion in ECs improves glucose homeostasis and insulin sensitivity in high-fat diet-fed mice. We next determined whether EC-CD36 loss affected FFAs and glucose in high-fat diet-fed
(HFD-fed) mice. EC CD36 deletion did not change BWs compared with those of controls (Figure 4A). As with chow, HFD-fed EC-Cd36-1 mice had higher circulating FFA levels (Figure 4B). These mice also had higher plasma TC and TG levels (Figure 4, $\mathrm{C}$ and D) due to increases in both LDL and HDL cholesterol (Supplemental Figure 5C). Total-body $\mathrm{C} d 36^{-/}$mice fed a HFD had improved insulin sensitivity (20). HFD-fed EC-Cd36 $6^{--}$mice had lower glucose and insulin concentrations (Figure 4, E and F) and improved OGTTs (Figure 4G) and ITTs (Figure 4H). Because we had seen such prominent changes in gene expression in the heart, we assessed insulin signaling after a fasting/feeding episode. After fasting and refeeding, EC-Cd36- hearts had greatly increased phosphorylation of AKT and the ribosomal protein $\mathrm{S} 6$ at S240/244, both of which are downstream targets of insulin signaling (Figures 4I), whereas heart insulin receptor (IR) phosphorylation did not change. To further assess insulin sensitivity, we obtained muscle, liver, and WAT from mice after insulin injection. In EC-Cd36-^ liver, we found that phosphorylated IR (p-IR) was significantly increased (Figure 4J). Deletion of Cd36 in ECs did not change IR, AKT, or S6 protein levels in skeletal muscle or WAT (Figure 4K and Supplemental Figure 5D). To test whether improved insulin sensitivity in HFD-fed EC-Cd36 $6^{--}$mice was 
A

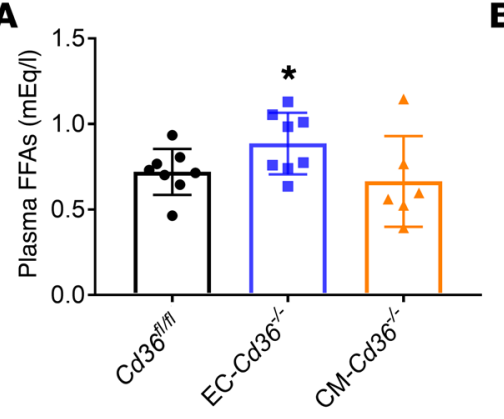

C

\begin{tabular}{|l|c|}
\hline & AUC $_{\text {TG (mg/dl720min) }}$ \\
\hline Cd36 $6^{\text {it/ft }}$ & $641.7 \pm 154.4$ \\
\hline EC-Cd36 & $1151.0 \pm 393.2^{*}$ \\
\hline CM-Cd36 & $574.6 \pm 238.2$ \\
\hline
\end{tabular}

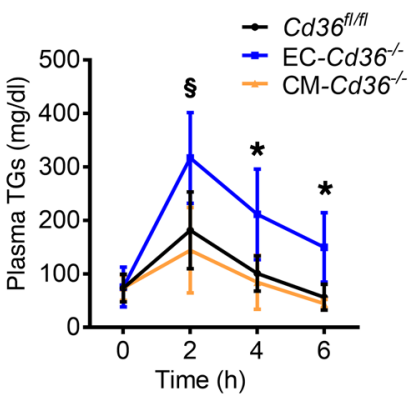

B

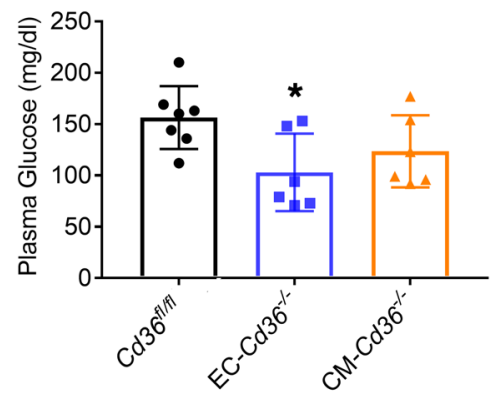

D

\begin{tabular}{|l|c|}
\hline & AUC $_{\text {OGTT (mg/d/1'120min) }}$ \\
\hline Cd36 & $2986.7 \pm 311.9$ \\
\hline EC-Cd36 & $2275.9 \pm 684.9^{*}$ \\
\hline CM-Cd36 & $2784.25 \pm 330.0$ \\
\hline
\end{tabular}

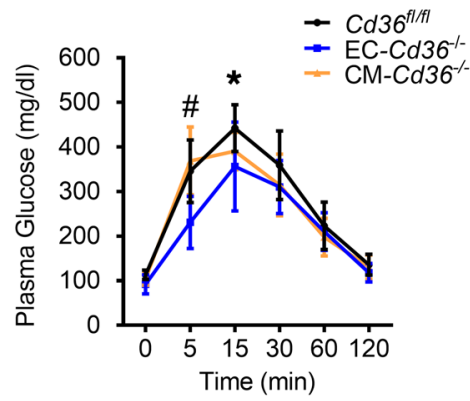

E

\begin{tabular}{|l|c|}
\hline & AUC $_{\text {ITT (mg/d/"120min) }}$ \\
\hline Cd36 & $1771.5 \pm 202.5$ \\
\hline EC-Cd36 & $1045.5 \pm 149.3^{*}$ \\
\hline
\end{tabular}

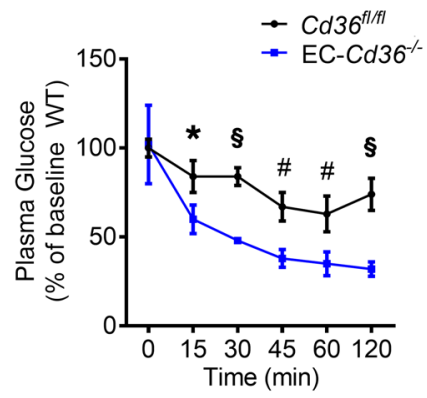

Figure 3. EC-Cd36-/- mice have increased FFA and postprandial TC levels but improved glucose metabolism. (A) Plasma FFAs in 4-month-old male mice fasted for 16 hours $(n=6-8)$. (B) Plasma glucose levels $(n=6-7)$ in $C d 36^{f / f f l}$, EC-Cd36 $6^{-1-}$, and CM-Cd36-/- mice. (C) Postprandial TC response in Cd36 $6^{f / / f l}$, $\mathrm{EC}-\mathrm{Cd} 36^{-/-}$, and $\mathrm{CM}-\mathrm{Cd}_{3} 6^{-/-}$mice. Plasma TC concentrations were measured $0,2,4$, and 6 hours after intragastric administration of olive oil $(n=5-7)$. Also shown is the AUC for postprandial TC levels. (D) Four-month-old male mice $(n=4-8)$ were fasted for 16 hours before oral glucose administration. Tail blood was collected for glucose measurements at $0,15,30,60$, and 120 minutes after glucose administration. Also shown is the AUC of glucose excursions. (E) For ITTs, mice were fasted for 4 hours and given $0.75 \mathrm{U} / \mathrm{kg} \mathrm{BW}$ insulin i.p. Tail blood samples were obtained before insulin administration $\left(t_{0}\right)$ and then 15 , $30,45,60$, and 120 minutes after insulin injection ( $n=4-5$ mice; data are representative of 2 independent experiments). Data represent the mean \pm SD. ${ }^{*} P<0.05,{ }^{\#} P<0.01$, and ${ }^{\circledR} P<0.001$ compared with $C d 36^{f / / f l}$ controls; 1-way ANOVA with Dunnett's multiple comparisons test.

related to reduced ceramides and diacylglycerols (DAGs), we assessed the distribution of these lipids in skeletal muscle and heart. Long-chain ceramides (Cer18:1) in the heart and very longchain ceramides (Cer22:0, Cer24:0, and Cer24:1) in both heart and skeletal muscle were significantly reduced in EC-Cd36 $\%$ mice (Figure 4, L and M). Cardiac and muscular DAGs were not altered (Supplemental Figure 5, E and F). Thus, EC-CD36 deletion protected mice from HFD-induced insulin resistance and was associated with reduced ceramide species.

CD36 deletion in ECs reduces LCFA uptake by heart, skeletal muscle, and BAT. To test directly whether loss of CD36 in ECs reduces the uptake of LCFAs in vivo, we injected $\left[{ }^{3} \mathrm{H}\right]$ oleic acid and $\left[{ }^{14} \mathrm{C}\right]$ hexanoic acid, which is not recognized by CD36, into 16-hourfasted male mice and assessed their tissue uptake. Compared with $C d 36^{A / f l}$ and $\mathrm{CM}-\mathrm{Cd} 36^{--}$mice, we observed that EC-Cd36 $6^{--}$mice had significantly delayed plasma clearance of oleic acid (Figure $5 \mathrm{~A}$ ) and a greater than $50 \%$ reduction in oleate uptake by heart, quadriceps muscle, and BAT (Figure 5B). In contrast, uptake by liver, lung, and kidney was unchanged. We observed similar results of reduced plasma LCFA clearance and uptake into heart and BAT in female mice (Supplemental Figure 6, A and B). However, skeletal muscle uptake was not altered in female mice. The lack of significant changes in skeletal muscle uptake in the female mice are consistent with the known sex differences in skeletal muscle lipid metabolism (21). The signal in WAT was low, and uptake differences were not significant. Hexanoic acid plasma removal and uptake into tissues were similar in mice of all 3 genotypes (Figure $5, C$ and D). These data show that EC-CD36 is required for optimal LCFA uptake by the heart, skeletal muscle, and BAT.

We also assessed acute LCFA accumulation in the heart and other tissues in real time using $\left[{ }^{11} \mathrm{C}\right]$ palmitate PET. As found with radiolabeled tracers, EC-Cd36-/ mouse hearts had a marked reduction in tracer uptake compared with $C d 36^{f / f l}$ and $\mathrm{CM}-\mathrm{C} d 36^{--}$ mouse hearts (Figure 5E). We also detected significantly reduced FA uptake by skeletal muscle and BAT in EC-Cd36-/ mice (Figure $5 F)$. In contrast to the reduction noted with EC deletion, neither $\left[{ }^{3} \mathrm{H}\right]$ oleic acid (Figure 5B) nor PET scan assessment of heart palmitate uptake (Figure 5E, right) in $\mathrm{CM}-\mathrm{Cd} 36^{-/-}$mice differed from what was observed in $C d 36^{\nexists f l}$ mice. Neither deletion affected palmitate uptake into the liver (Supplemental Figure 6C).

CD36 deletion in ECs increases glucose uptake by heart, skeletal muscle, and BAT. Fasting reduces glucose uptake by heart and muscle, an adaptation impaired by total CD36 deficiency in humans $(22)$ and mice $(4,9)$. We examined tissue uptake of $\left[{ }^{18} \mathrm{~F}\right]$ 
A

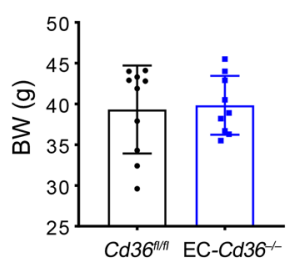

G

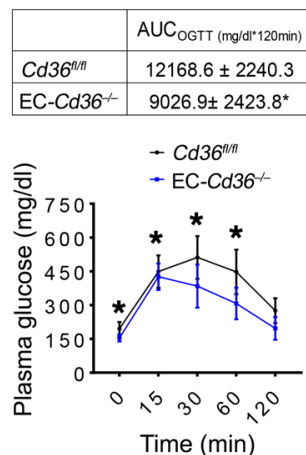

$\mathbf{L}$

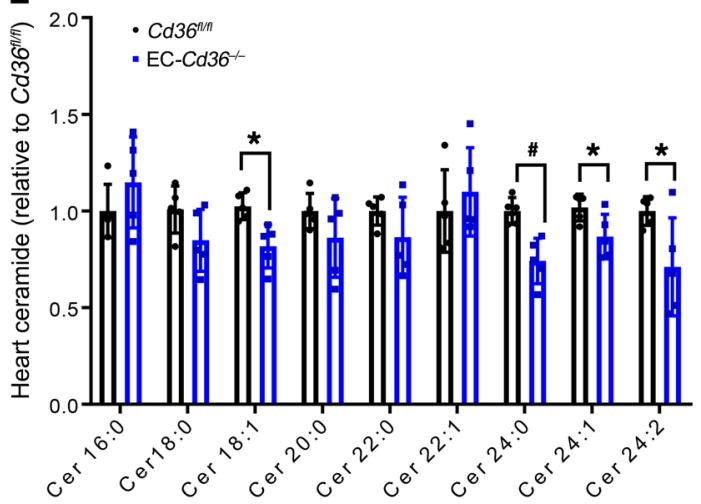

C

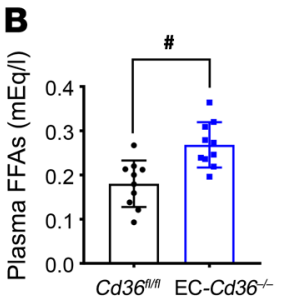

$\mathbf{H}$
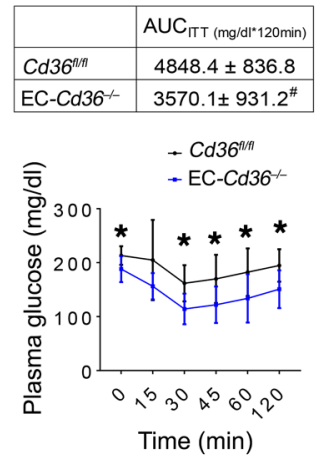
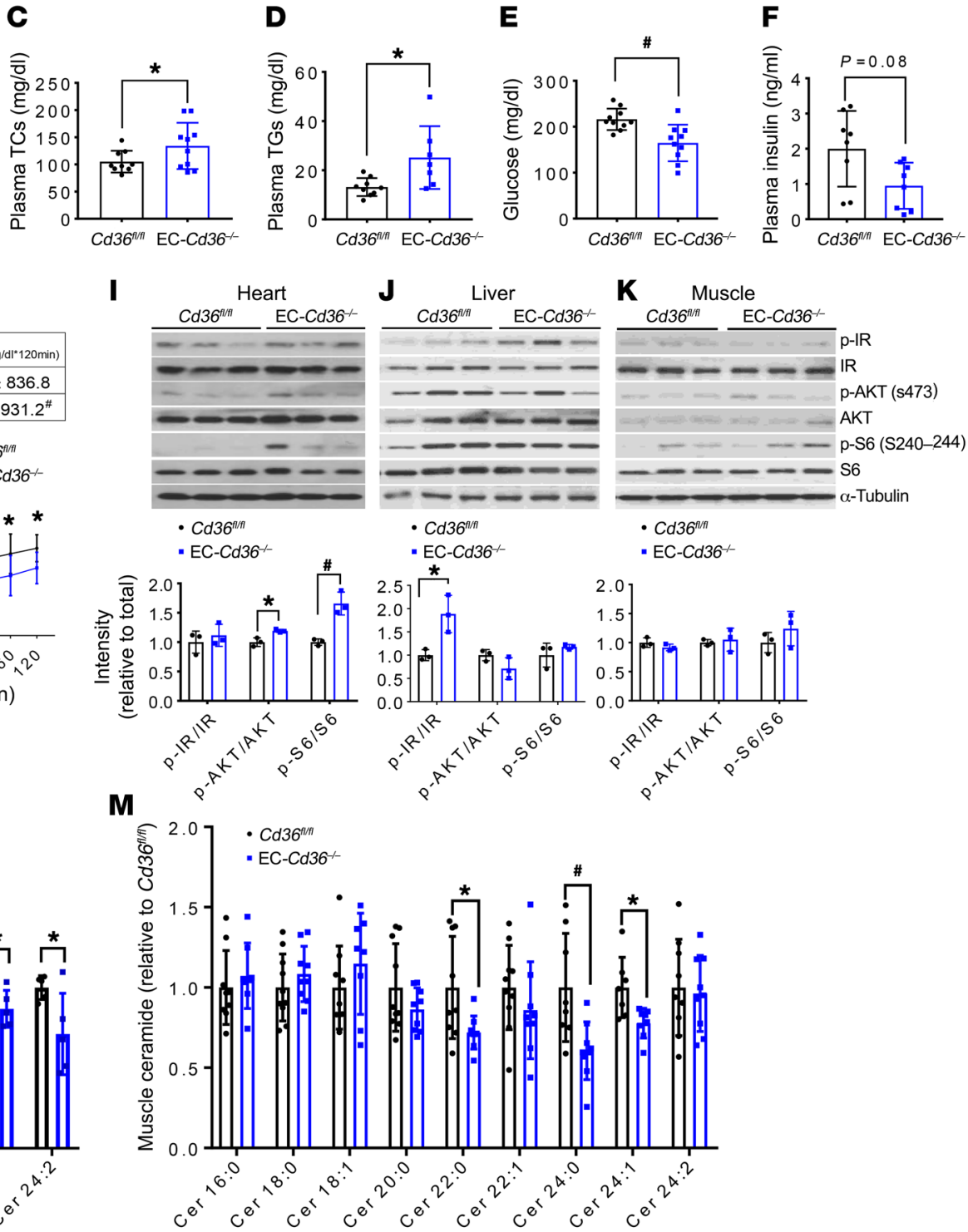

Figure 4. EC-Cd36-/- improves glucose homeostasis and increases insulin sensitivity in 6-month-old male mice fed a HFD for 4 months. (A-E) BW, plasma lipids, and glucose after a 16-hour fast. (F) Plasma insulin after a 16-hour fast followed by a 4-hour refeeding period. $P$ value was determined by 1 -way ANOVA. (C) For GTTs, male mice fasted for 16 hours were given oral glucose, and tail blood samples were collected for glucose measurements at $0,15,30$, 60, and 120 minutes. Also shown is the AUC of glucose excursions. (H) For ITTs, mice fasted for 4 hours were given $0.75 \mathrm{U} / \mathrm{kg}$ BW insulin i.p. Tail blood samples were obtained at $t_{0}$ and then $15,30,45,60$ and 120 minutes after insulin administration. (I) Activity of IR, AKT, and S6 in Cd36 fl/fl and EC-Cd36 ${ }^{-1-}$ mice 20 minutes after insulin injection, with densitometric analysis of (I) heart, (J) liver, and (K) skeletal muscle tissue. (L and $\mathbf{M})$ Individual ceramide (Cer) species in heart $(n=5)$ and muscle $(n=9-10)$. Data represent the mean \pm SD. ${ }^{*} P<0.05$ and ${ }^{\#} P<0.01$ compared with Cd36 $f^{f / f l}$ controls; 1 -way ANOVA with Dunnett's multiple comparisons test (A-E and $\mathbf{G}-\mathbf{M})$.

deoxyglucose (FDG]DG) using PET in 16-hour-fasted mice and found that glucose uptake increased in heart, muscle, and WAT of EC-Cd36-- mice compared with controls (Figure 5G). In contrast, tissues of $\mathrm{CM}-\mathrm{Cd} 36^{--}$mice showed no changes in glucose uptake (Supplemental Figure 6D). These data show that EC CD36 regulates the fasting-induced metabolic shift by tissues, which spares glucose use and increases reliance on FAs.

CD36 deletion in either ECs or CMs reduces heart lipid storage. The heart normally increases its lipid stores during fasting. Studies in $\mathrm{Cd}_{36^{-/}}$mice suggest that $\mathrm{CD} 36$ is required for this adaptation $(4,9,10,23)$. We fasted mice for 16 hours and assessed lipid droplet accumulation using BODIPY dye. We found that lipid droplet accumulation was significantly reduced in EC-C $d 36^{-1-}$ hearts (Figure 6A). Lipid droplet numbers were significantly decreased in both EC-Cd36-- and CM-Cd36 $6^{-1-}$ hearts, as assessed by electron microscopy (Figure 6, B and C), while heart morphology appeared normal. Direct measurements of heart TGs showed that hearts from EC-Cd36-/ mice had a $49 \%$ reduction in TGs and $\mathrm{CM}-\mathrm{Cd} 36^{--}$mice had a 53\% reduction (Figure 6D). Thus, CD36 deletion in either ECs or CMs reduced heart lipid accumulation. We also observed decreased cardiac lipid accumulation in VE-Cd36 $6^{--}$mice (Supplemental Figure 7, A and B). As might be expected with reduced lipid uptake into peripheral tissues, EC-Cd36-/ mice had increased liver TG content (Supple- 
A

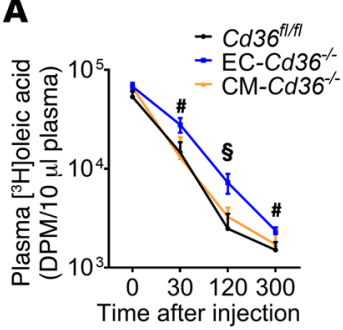

(s)
B

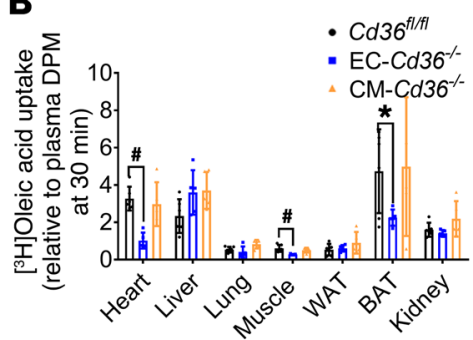

C

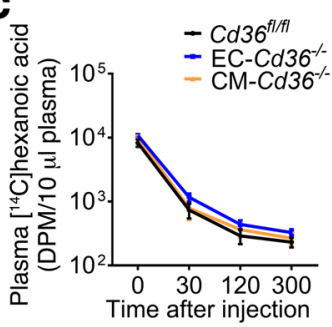

(s)

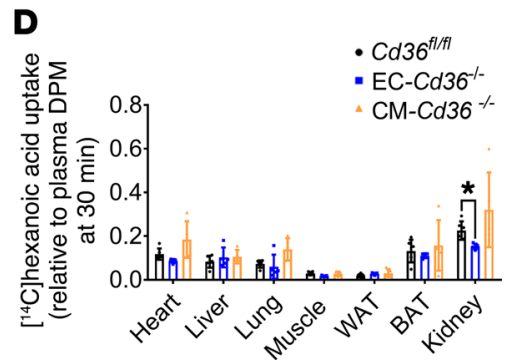

E

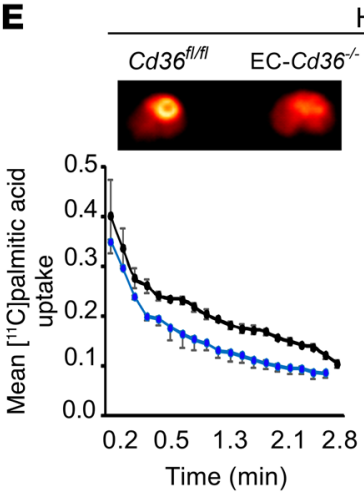

Heart
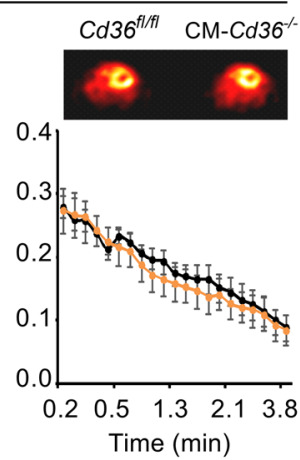

$\mathbf{F}$

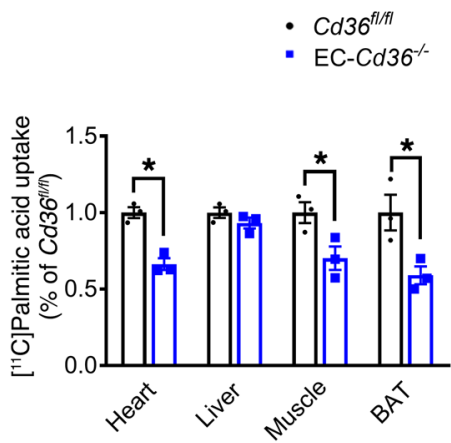

G

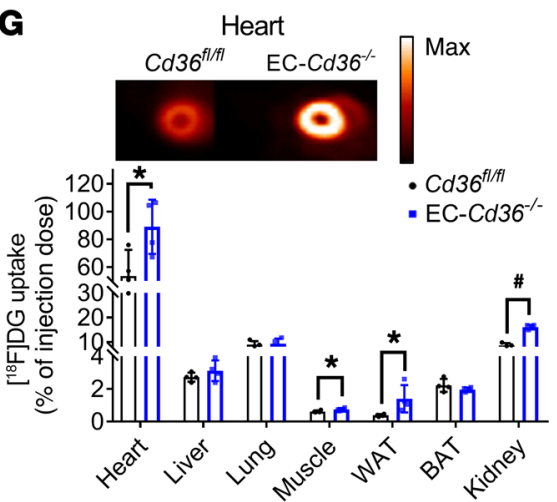

Figure 5. EC-Cd36-/- mice have reduced tissue LCFA uptake during fasting. (A-D) Uptake of oleic and hexanoic acids in EC-Cd36-/- mice. Four-month-old mice were fasted for sixteen hours and given a tail i.v. injection of [ $\left.{ }^{3} \mathrm{H}\right]$ oleic acid and $\left[{ }^{14} \mathrm{C}\right]$ hexanoic acid. (A) Plasma ${ }^{3} \mathrm{H}$ radioactivity in $\mathrm{Cd} 36^{f / / f l}$, EC-Cd36 $6^{-1-}$, and $\mathrm{CM}-\mathrm{Cd} 36^{-/-}$mice $(n=5-6)$ measured at the indicated time points after injection. Scale bars: $50 \mu \mathrm{m}$. (B) ${ }^{3} \mathrm{H}$ content of different tissues, (C) plasma ${ }^{14} \mathrm{C}$ radioactiv-

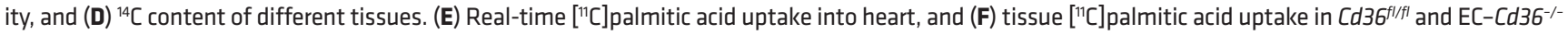
male mice $(n=4)$. (G) Tissue FDC uptake in $C d 36^{f / f l}$ and $E C-C d 36^{-/-}$mice $(n=4)$ derived from summing the uptake kinetics up to 60 minutes after tracer administration. Also shown are representative scans of FDG accumulation at 60 minutes in the hearts of control and EC-Cd36-- 16-hour-fasted mice. Max, maximum. Data represent the mean $\pm \mathrm{SD}$. ${ }^{*} P<0.05,{ }^{\#} P<0.01$, and ${ }^{\S} P<0.001$ compared with $C d 36^{f / / f l}$ controls; 1-way ANOVA with Dunnett's multiple comparisons test.

mental Figure 7C). The increase in heart TGs was associated with an increase in diacylglycerol acyl transferase 1 (DGAT1) (Supplemental Figure 1E), a compensation that might have prevented the accumulation of lipids that interfere with insulin signaling $(24,25)$.

EC or CM CD36 deletion reduces the expression of FA metabolism heart genes and differentially alters glucose metabolism genes. We performed RNA sequencing (RNA-seq) to gain an unbiased global view of transcript expression in EC-Cd36 $6^{-/}$and $\mathrm{CM}-\mathrm{Cd} 36^{-/-}$ mouse hearts. Gene heatmap analysis (Figure 7) showed marked expression changes in hearts from both groups of mice. Expression of glucose metabolism and glucose signaling genes showed differential regulation. In EC-Cd36/- mouse hearts, we detected increases in mRNA levels of genes mediating glucose uptake and oxidation such as Slc2a1 (Glut1), Slc2a4 (Glut4), Pcx (pyruvate carboxylase), Hk2 (hexokinase 2), Gck (glucokinase), and $P f k$ fb2 (6-phosphofructo-2-kinase/fructose-2,6-biphosphatase 2). Decreased expression of $P d k 4$ (pyruvate dehydrogenase kinase 4), which inhibits pyruvate conversion to acetyl-CoA, was consistent with enhanced glucose utilization. These changes were associated with increased expression of insulin signaling pathway genes such as Irs1 (insulin receptor substrate 1), Pten (phosphatase and tensin homolog), Pik3r1 (phosphoinositide 3 kinase regulatory subunit 1 , also known as p85), Foxo1 (Fox protein 01), and Mapk4 (MAPK4).

In $\mathrm{CM}-\mathrm{Cd} 36^{-/-}$mice, in contrast to $\mathrm{EC}-\mathrm{Cd} 36^{-/-}$mice, we detected reduced cardiac expression of glucose metabolism genes, while expression of genes for insulin signaling was not affected. These data suggest that CD36 deletion in ECs enhances the expression of genes related to glucose utilization and insulin action, while the effect of the deletion in CMs is opposite or neutral.

PPARs, especially PPAR $\alpha$, modulate LCFA oxidation in the heart. Ppara mRNA levels increased in EC-Cd36-/- hearts (Figure 7, red arrow), while levels of PPAR $\gamma$ and PPAR $\beta$, and PPAR $\delta$ were unchanged in both EC- and CM-KO hearts. However, expression of FA metabolism genes including many PPAR $\alpha$ targets was decreased in both groups of mice. For example, expression of Slc27a1 (FA transport protein 1), Cpt1a (carnitine palmitoyl transferase 1a), Cpt2, Acot2 (acyl CoA thioesterase 2), Acadl (acyl CoA hydrogenase 1), Acadm (medium chain ACAD), and Hsd17b4 (hydroxysteroid $17 \beta$ dehydrogenase 4 ) decreased markedly in EC-Cd36 ${ }^{-/}$and CM-Cd36 $6^{-/-}$hearts. In both groups, Slc25a2O (carnitine acyl carnitine translocase, also known as $\mathrm{Cact}$ ), which mediates the transport of acylcarnitines from the cytosol to the mitochondrial matrix, was reduced (Figure 7). These changes indicate that CD36 in both ECs and CMs regulates tissue FA metabolism. In $\mathrm{CM}-\mathrm{Cd} 36^{-/}$hearts, these changes were consistent with the effects of CD36 on FA oxidation, as documented in an overexpression mouse model (26) and in tissue culture experiments (27).

Kyoto Encyclopedia of Genes and Genomes (KEGG) analyses of insulin- and PPAR-activated pathways highlighted the enhanced insulin signaling detected in EC-Cd36/- hearts, with increased expression of major regulators of GLUT4 translocation, lipogenesis, and glycogenesis (Figure 8A). This was not apparent in $\mathrm{CM}-\mathrm{Cd} 36^{--}$hearts. We noted diminished expression of major PPAR targets in EC-Cd36 $6^{-/-}$hearts (Figure 8B). Expression levels 
A

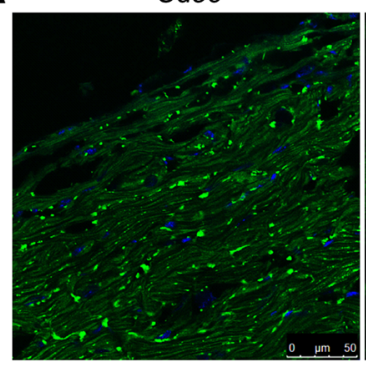

B

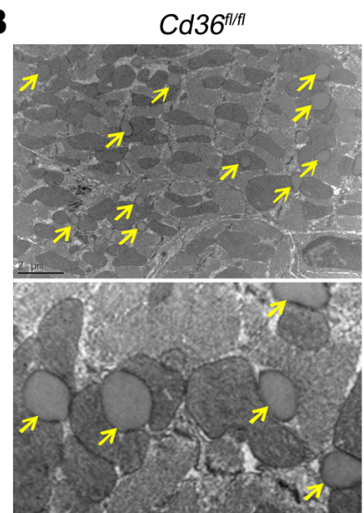

C

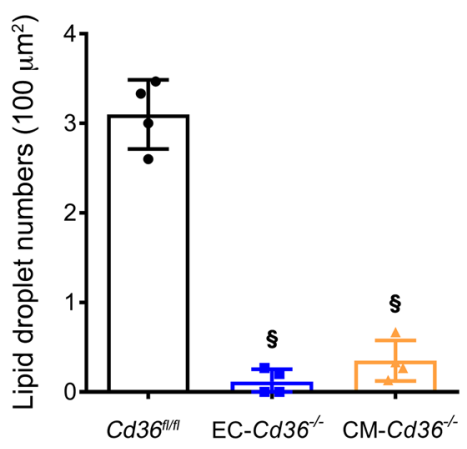

$\mathrm{EC}-\mathrm{Cd} 36^{-1-}$

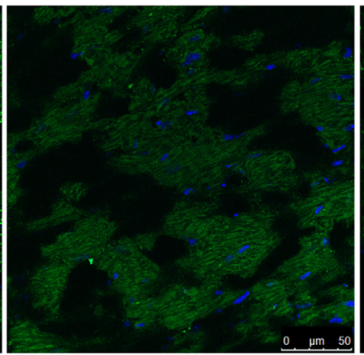

$\mathrm{EC}-\mathrm{Cd} 36^{--}$

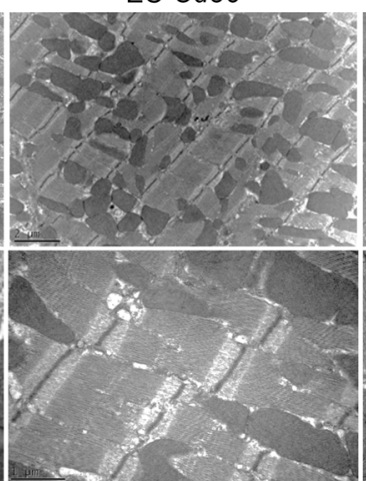

D

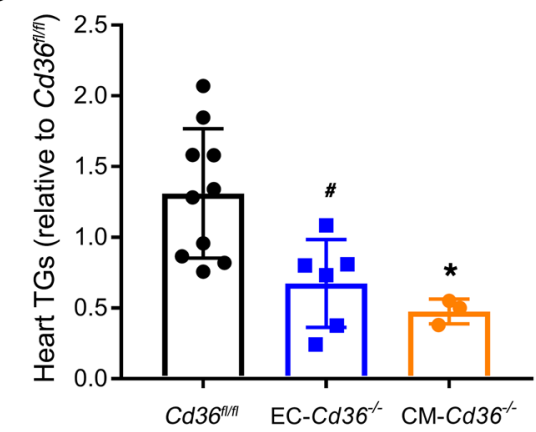

Figure 6. CD36 deletion in ECs or CMs reduces heart lipid storage during fasting. (A) Heart tissue sections from $C d 36^{f l / f I}$, EC-Cd36 $6^{-1-}$, and CM-Cd36-1- mice were stained with BODIPY 493/503. Scale bars: $50 \mu \mathrm{m}$. (B) Electron micrographs (original magnification $\times 3,400$, top row, and $\times 15,000$, bottom row) of myocardial tissue showing a significant decrease in lipid droplets within the sarcoplasm of CMs from EC-Cd36-/- and CM-Cd36mice. Yellow arrows indicate lipid droplets. (C) Lipid droplet numbers in heart tissue sections $(n=4)$ from $C d 36^{f l / f l}, \mathrm{EC}-\mathrm{Cd} 36^{-/-}$, and CM-Cd36-/- mice (expressed per $\left.100 \mu \mathrm{m}^{2}\right)$. (D) Heart TC content in $C d 36^{f / f l}(n=10)$, $\mathrm{EC}-\mathrm{Cd} 36^{-/-}(n=6)$, and CM-Cd36- mice $(n=3)$. Data represent the mean \pm SD. ${ }^{*} P<0.05,{ }^{\#} P<0.01$, and $\$ P<0.001$ compared with $C d 36^{f / / f l}$ controls; 1 -way ANOVA with Dunnett's multiple comparisons test. of several PPAR and insulin signaling pathway genes such as Pparo and Glut1 were increased in hearts of EC-Cd36/- mice, while Fas and Ampk expression levels were decreased (Figure $8 \mathrm{C}$ ). Hearts from $\mathrm{CM}-\mathrm{Cd} 36^{-/-}$mice, like those from germline $C d 36^{-/-}$mice, had higher Ampk mRNA levels (Supplemental Figure 8A) (10, 23). This contrasted with lower Ampk levels in hearts from EC-Cd36-/- mice (Figure 8A), perhaps reflecting greater glucose uptake. However, like in EC-Cd36 ${ }^{-/}$hearts, we found that expression levels of major PPAR target genes in $\mathrm{CM}-\mathrm{Cd} 36^{-/-}$hearts were decreased (Supplemental Figure $8 \mathrm{~B}$ ). These data were confirmed by qRT-PCR (Figure $8 \mathrm{C}$ ). We also observed decreased expression of Pdk4, Cpt1, and Aox mRNA in female EC-Cd36 $6^{--}$mouse hearts (Supplemental Figure 8C).

\section{Discussion}

Delivery of LCFA to parenchymal cells in tissues that do not have fenestrated capillaries involves transfer from the circulation to parenchymal cells. This movement of LCFAs requires their transfer either through or around ECs. CD36 has been reported to mediate LFCA uptake into muscle and adipose tissues $(11,12)$ and LCFA accumulation in CMs (28) and other cells (29), but its role in ECs had not been directly studied. We show that ECs robustly express
CD36 and that EC-specific CD36 deletion reduces acute LCFA uptake into heart and other tissues. Moreover, EC-Cd36 ${ }^{--}$mice have increased insulin sensitivity and lack myocardial lipid droplet accumulation with fasting. Reductions in ceramides might have contributed to the improved insulin sensitivity. The reduction of FA uptake into BAT might have important implications for thermogenesis. In this regard, Bartelt et al. (30) have shown that both LpL and CD36 affect BAT uptake of FAs during cold exposure.

Several reports have suggested that ECs are a major regulator of tissue LCFA metabolism. These reports did not directly investigate EC CD36, but some included indirect evidence that is consistent with our current findings. Deletion of EC PPAR $\gamma$ increased circulating LCFAs (31) and reduced expression of several PPAR $\gamma$ gene targets in ECs, including CD36. Meox2/Tcf15 heterodimer transcription factors (TFs) in ECs have been reported to regulate FA transfer across the cardiac capillary endothelium (32) and to affect the expression of FA-metabolizing genes, including $C d 36$. Deletion of EC VEGF-B (33) was reported to cause changes in EC LCFA uptake as a result of transcriptional regulation of vascular-specific FA transport proteins 3 and 4, but this was not confirmed by others (34). A recent report showed that a metabolite of branch chain ami- 


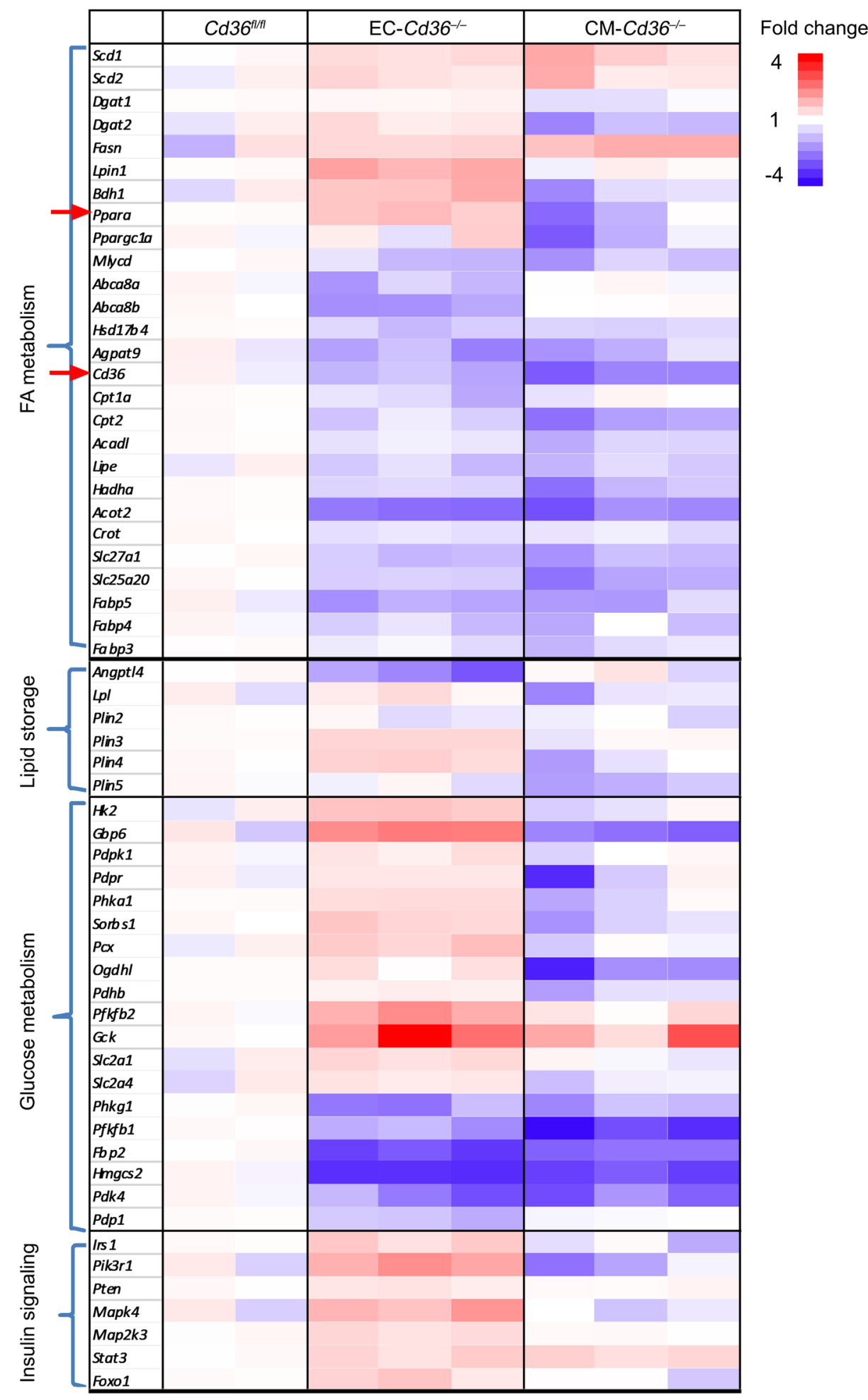

Figure 7. Heart tissue mRNA expression in mice with EC or CM CD36 deletion. Clustered heatmaps from RNA-seq data showing differential expression profiles for $C d 36^{f / / f l}$, EC-Cd36 $6^{-/}$, and $\mathrm{CM}-\mathrm{Cd} 36^{-/-}$mice. Red indicates overabundant expression; blue indicates underabundant expression.

no acids modulated both LCFA movement across ECs and insulin sensitivity (35). Interestingly, the molecular mechanisms mediating FA uptake by EC CD36 might differ among tissues. Salameh et al. recently reported on a mechanism specific to adipocytes, describing a prohibitin-annexin A2 complex that increases CD36 on the cell surface and FA uptake by ECs and adipocytes (36).

Robust CD36 expression in capillary ECs (37) is found in tissues that are most active in circulating LCFA extraction and that also express LpL and its EC anchoring protein glycosylphosphatidylinositol-anchored high-density lipoprotein binding protein 1 (GPIHBP1). This expression pattern suggests that $\mathrm{CD} 36$ is important in LCFA trafficking across smaller blood vessels and capillaries, where most nutrient uptake occurs. ECs are not major sites of FA oxidation, so CD36 in capillaries is likely to be more important for LCFA transport than for LCFA-derived ATP production. This conclusion is supported by our data showing that lack of CD36 expression does not change EC mRNA levels of FA or glucose metabolism genes.

The lack of lipid droplet accumulation with EC and CM CD36 deletion shows that CD36 expression at both sites is required for optimal delivery of LCFAs to the heart. The defect in uptake into EC-Cd36/- mouse hearts was evident using 2 types of tracer kinetic studies: radiolabeled LCFA and $\left[{ }^{11} \mathrm{C}\right]$ palmitate PET. Both of these techniques assess the rapid accumulation of LCFAs and, for this reason, might have failed to show a defect in LCFA uptake into $\mathrm{CM}-\mathrm{C} d 36^{--}$hearts; $\mathrm{CM}$ CD36 is well established as a mediator of LCFA accumulation in cultured CMs $(38,39)$ and as a provider of substrates required for the response to acute afterload (40). In addition, our studies highlight important differences in the contribution of ECs versus CMs to FA metabolism in the heart, and additional studies are needed to understand the mechanisms underlying these differences.

Loss of EC CD36 led to improved insulin action and glucose disposal, which we demonstrated using cardiac gene expression, OGTTs, and ITTs. These effects were confirmed in HFDfed mice. Improvements in OGTTs and ITTs were similar to those observed with PPAR $\alpha$ (41) or GLUT4 deletion (42). In addition, circulating glucose levels were reduced, as was found with some (43), but not all (44), genetic mutations that reduce FA oxidation. FDG PET showed increased glucose uptake into heart, skeletal muscle, and WAT with EC CD36 deletion. CM-Cd36-/- mice did not have sim- 


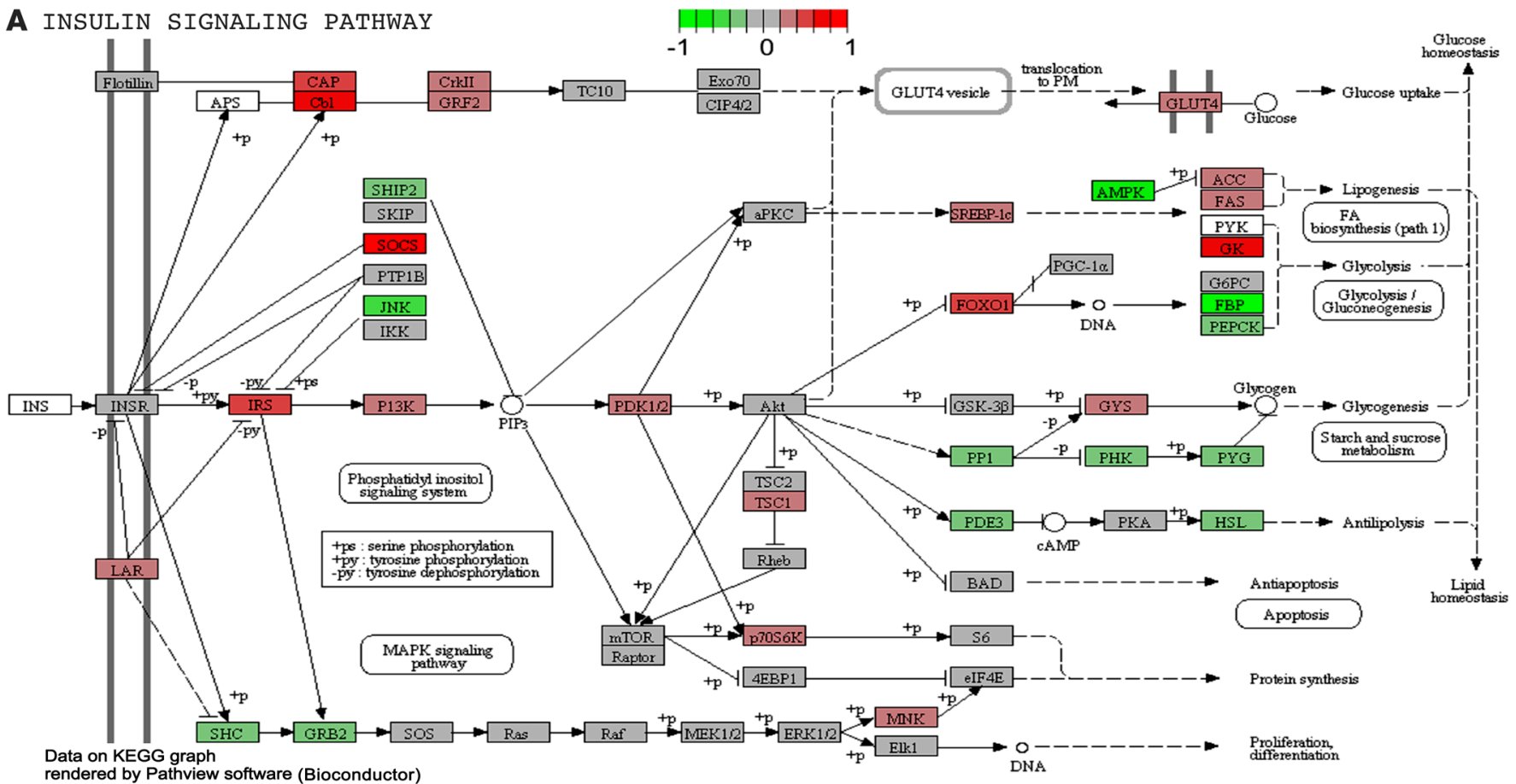

B PPAR SIGNALING PATHWAY
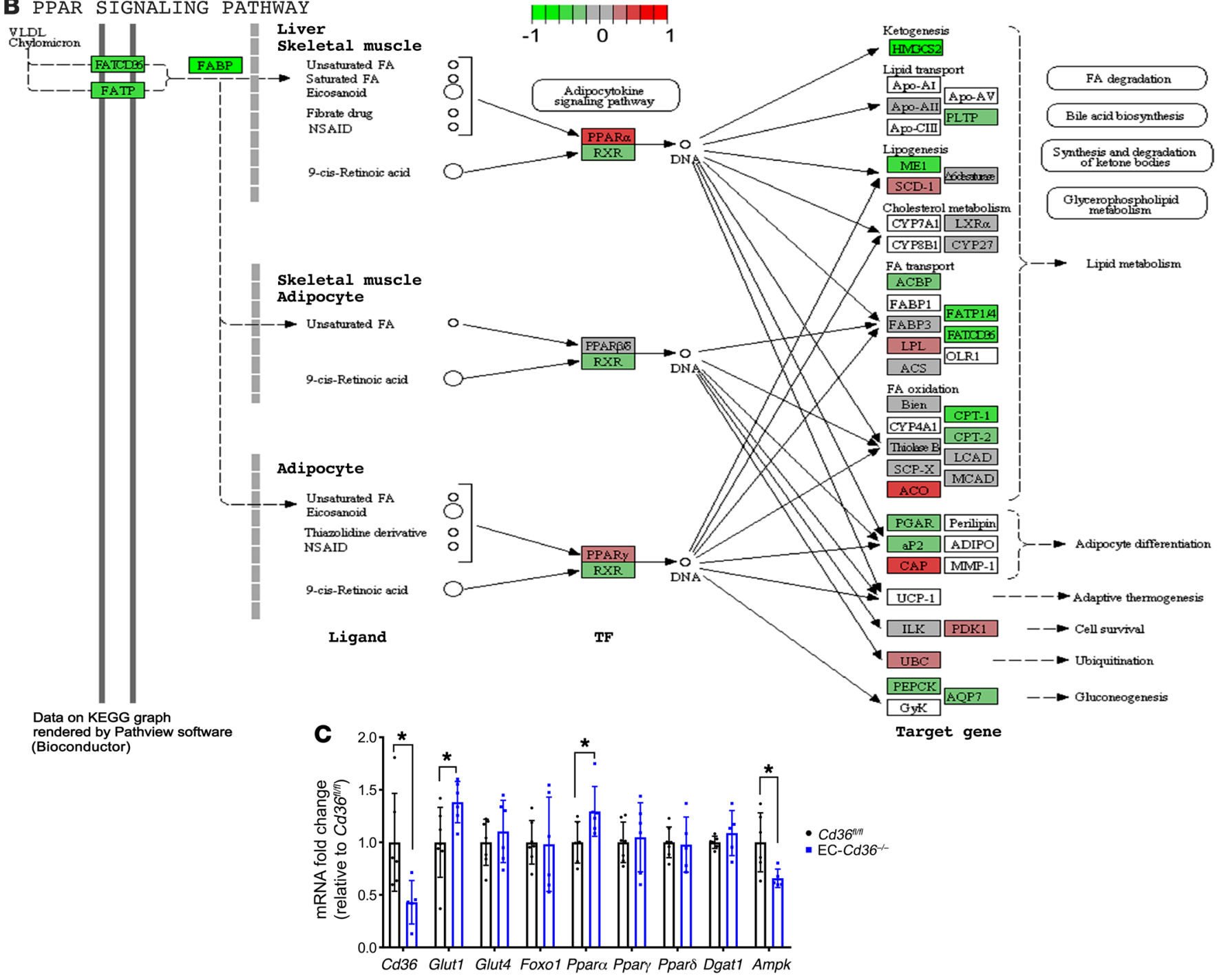
Figure 8. KEGG analysis of insulin signaling and PPAR pathways. KEGC analysis of (A) insulin and (B) PPAR signaling pathways. (C) qRT-PCR analysis of heart mRNA in $\mathrm{Cd} 36^{f / / f l}, \mathrm{EC}-\mathrm{Cd} 36^{-/-}$, and $\mathrm{CM}-\mathrm{Cd} 36^{-/-}$mice $(n=$ 5-7). Data are shown as the mean $\pm \mathrm{SD} .{ }^{*} P<0.05$ compared with $C d 36^{f / / f l}$ controls; 1-way ANOVA with Dunnett's multiple comparisons test.

ilar improvements in insulin action or glucose metabolism, and, instead, hearts from these mice had downregulated expression of some glucose uptake genes, opposite to what would be expected with reduced lipid uptake. These changes might reflect myocyte-autonomous actions of CD36 that regulate insulin signaling (45). These separate effects on parenchymal biology could explain the propensity to develop insulin resistance in humans with CD36 mutations (46).

In humans, CD36 deficiency has a prevalence of $0.3 \%$ to 9\%, with a higher incidence in Asian and African populations. Individuals with complete CD36 deficiency have higher serum levels of fasting TGs (47) and chylomicron remnants (19). These lipid abnormalities likely reflect defective tissue FA metabolism, as CD36-deficient individuals show markedly reduced LCFA uptake by the heart $(14,22)$ and have enhanced myocardial glucose uptake (22).

In summary, our data show that ECs are major sites of regulation of LCFA uptake and, in turn, tissue lipid metabolism and insulin sensitivity. By regulating heart and skeletal muscle FA delivery and glucose utilization, EC CD36 is a major factor affecting tissue fuel selection and systemic metabolism. Whether EC CD36 actions are beneficial for normal or diseased hearts is uncertain (48-50) and probably depends on the physiologic situation. Reduction of LCFA uptake leads to impaired energy production during fasting or exercise, but also protects against excess lipid accumulation (lipotoxicity) (51) and improve glucose utilization during hypoxia (52). The vascular endothelium represents an important and accessible therapeutic target (53), and our findings suggest that targeting EC CD36 could be a means to influence tissue FA uptake and prevent or treat a variety of diseases caused by abnormal lipid utilization.

\section{Methods}

Generation of $C d 36^{f / f l}$ mice. A Lox (L83) site and Frt-Neo-Frt-LoxP (FNFL) cassette were engineered to flank a $1.7-\mathrm{kb}$ sequence with exons 3 and 4 of CD36 to generate a floxed/Neo CD36 allele on a bacterial artificial chromosome (BAC). A gene-targeting vector was created by retrieving the 2-kb short homology arm ( $5^{\prime}$ to L83), the floxed sequence containing exons 3 and 4, the FNFL cassette, and the 10-kb-long homology arm (end of FNFL to $3^{\prime}$ ) into a plasmid vector carrying a diphtheria toxin $\alpha$ (DTA) chain negative selection marker. The FNFL cassette conferred G418 resistance during gene targeting in KV1 (129B6 hybrid) embryonic stem (ES) cells, and the DTA cassette provided autonomous negative selection to reduce random integration. Identified targeted ES cells were injected into C57BL/6 blastocysts to generate chimeric mice. Male chimeras were bred with homozygous ACTB (Flpe/Flpe) C57BL/6 females to remove the neomycin cassette and transmit the floxed CD36 allele (Flpe recombinase). The mice were then crosLoxsed with Tie2-Cre and MHCCre mice (C57BL/6) to generate EC-Cd36 ${ }^{-/-}$and $\mathrm{CM}-\mathrm{Cd} 36^{-/-}$mice and with LysM-Cre mice to create myeloid cell deficiencies. Found- er $C d 36^{f / / A}$ mice were identified by tail genomic DNA analysis with primer $1 \mathrm{~F}$ specific to the upstream LoxP locus (5'-ATTGGCATCTGTGTAGCGCTCTTGGC-3') and primer 1R (5'-TGCTACTATGCACTCCATGCAGGC-3'), an antisense specific to the downstream LoxP locus. CD36 deletion in EC-Cd36 $6^{-/-}$hearts was confirmed by PCR of heart tissue DNA and mRNA with primers 2 and 3 (Supplemental Table 1), respectively. Mice were maintained under a constant light-dark cycle (light from 7:00 am to 7:00 pm) and received either a standard diet (catalog 5053, LabDiet) or a HFD containing $65 \%$ fat (catalog D12492, Research Diets).

Human tissues. Normal human heart tissues were obtained from the Department of Surgery at Columbia University Medical Center.

Western blot analysis. Total proteins were obtained from fresh heart tissues of 4- to 6-month-old EC-Cd36/- and CM-Cd36 $6^{--}$mice and littermate controls using a RIPA Kit (Santa Cruz Biotechnology). Proteins $(30 \mu \mathrm{g})$, separated by Western blotting, were probed for CD36 (catalog AF2519, R\&D Systems, 1:200 dilution) and $\alpha$-tubulin (catalog ab7291, Abcam, 1:5,000 dilution) as a control. Antibodies against Akt (Ab 9272), p-Akt (S473) (mAb 4060), S6 (mAb 2217), p-S6 (S240/244) (Ab 2215), IR (mAb 3025), p-IR (mAb 3024), and $\beta$-actin (mAb 4970) were obtained from Cell Signaling Technology. Signals were quantified by densitometry using the Odyssey Fc Imaging System (LI-COR Biotechnology).

Heart and plasma lipids. Blood from fasted (4 or $16 \mathrm{~h}$ ) mice was collected from the retro-orbital plexus. Plasma TC, TGs, and FFA were measured enzymatically using an Infinity Kit (Thermo Electron Corp.) and a NEFA C Kit (Wako). To measure heart TGs, hearts were perfused with PBS and homogenized at $4^{\circ} \mathrm{C}$ in $1 \mathrm{M} \mathrm{NaCl}$ buffer with lipase inhibitors. Lipids were extracted from heart tissues $(50 \mathrm{mg}$ ) according to a modified Folch method (54). The dried lipids were solubilized in PBS with $2 \%$ Triton $\mathrm{X}-100$, and TG levels were measured using an Infinity TG Kit (Thermo Electron, Thermo Fisher Scientific.).

IHC. Human and mouse cardiac ventricular specimens were fixed in $10 \%$ buffered formaldehyde and embedded in paraffin, and 5 - $\mu \mathrm{m}$-thick microtomic sections were stained with a 1:100 dilution of mouse and human CD36 antibodies (AF2519, AF1955; R\&D Systems). After washing, sections were incubated ( $2 \mathrm{~h}$ at room temperature) with a biotinylated rabbit anti-goat IgG antibody. Staining specificity was tested by omission of primary antibodies.

Immunofluorescence staining. Cardiac ventricular tissues from 4-month-old male mice were embedded in Tissue-Tek OCT Compound (Sakura). Frozen sections (5- $\mu$ m thickness) were fixed in 10\% formalin for 30 minutes, washed 3 times in PBS, and blocked for 1 hour in $1 \%$ BSA-PBS at room temperature. The fixed sections were incubated ( $2 \mathrm{~h}$, room temperature) with a 1:100 dilution of either mouse CD36 (catalog AF2519, R\&D Systems) or CD31 (catalog 102514, BioLegend) antibody followed by washing and incubations $(1 \mathrm{~h})$ with a secondary antibody (1:500 dilution) coupled to Alexa Fluor 488 or 594 (Invitrogen, Thermo Fisher Scientific). Washed sections were then stained with ProLong Gold Antifade Mountant with DAPI (Thermo Fisher Scientific), and digital images were obtained with a Leica confocal microscope. Isolated ECs were fixed with $4 \%$ PFA, permeabilized with $0.2 \%$ Triton $\mathrm{X}-100$, and blocked with donkey serum. Then, cells were incubated with anti-VE-cadherin (ab33168, Abcam) and CD36 (AF2519, R\&D Systems) antibodies followed by secondary antibodies conjugated with Alexa Fluor 488 and Alexa Fluor 594. Nuclei were visualized with DAPI. Imaging was performed using a Nikon Eclipse inverted microscope at $\times 60$ magnification. 
Lipoprotein isolation. Plasma was pooled from EC-Cd36-/- mice fed chow or a HFD. Lipoprotein fractions were separated by a previously described method (55).

OGTT. $C d 36^{f / f l}, \mathrm{EC}-\mathrm{Cd} 36^{-/}$, and CM-Cd36-/- mice fasted for 16 hours were given $2 \mathrm{~g}$ glucose $/ \mathrm{kg}$ body mass intragastrically. Tail blood samples were collected and glucose measured 0, 15, 30, 60, and 120 minutes after gavage.

Plasma insulin measurements. $\mathrm{Cd} 36^{f / f l}$ and EC-Cd36 $6^{-/-}$were fasted for 16 hours and refed for 2 hours. Blood was collected from the retro-orbital plexus, and plasma insulin levels were measured using a mouse ultrasensitive insulin ELISA kit (ALPCO).

ITTs. Mice were fasted for 4 hours and then given human insulin (Eli Lilly, $0.75 \mathrm{U} / \mathrm{kg} \mathrm{BW)} \mathrm{intraperitoneally.} \mathrm{Tail} \mathrm{blood} \mathrm{was} \mathrm{obtained} \mathrm{0,}$ $15,30,45,60$, and 120 minutes after insulin injection.

Insulin signaling test. For insulin-induced changes, 2 different methods were used to assess insulin signaling in 3-month-old male mice fed a HFD for 6 weeks using. Changes in the heart were most evident when assessed after fasting (16 h) followed by refeeding ( $2 \mathrm{~h}$ ), after which hearts were harvested and used for Western blot analysis. Other tissues were studied after i.p. insulin injection (0.50 U/kg BW human insulin, Eli Lilly). Skeletal muscle, liver, and WAT were harvested 20 minutes after insulin injection and then used for Western blotting.

Lipid droplet staining. Cardiac ventricular tissues from 4-monthold male mice were embedded in Tissue-Tek OCT Compound (Sakura). Frozen sections of myocardium (5- $\mu$ m thickness) were fixed in $10 \%$ formalin for 30 minutes at room temperature and then washed 3 times in PBS. The fixed sections were then incubated with $4 \mu \mathrm{M}$ BODIPY 493/503 dye (catalog D3922, Invitrogen, Thermo Fisher Scientific) for 30 minutes at room temperature. After washing, sections were stained with ProLong Gold Antifade Mountant with DAPI (Thermo Fisher Scientific) and covered with glass coverslips (VWR, Avantor). The digital images were obtained with a Leica confocal microscope.

Postprandial lipemia. After a 16-hour fast, mice maintained on a chow diet received an intragastric bolus of $0.3 \mathrm{ml}$ olive oil. Tail blood samples were collected before and 2, 4, and 6 hours after gavage. Plasma TG concentration was assayed with an Infinity Kit (Thermo Fisher Scientific).

EC isolation. Lungs harvested from $\mathrm{Cd} 36^{f / / f l}$ and EC-Cd36-1mice (Tie2-Cre) were minced and enzymatically digested in MACS C-tube (Miltenyi Biotec). Cells were centrifuged, resuspended in PEB buffer (1× PBS, 0.5\% BSA, 2 mM EDTA), blocked with FcR blocking reagent (Miltenyi Biotec), and then incubated with CD31 conjugated microbeads for 15 minutes at $4^{\circ} \mathrm{C}$. The cell suspension was passed through a MACS separation column, the column was washed with PEB buffer $(3 \times)$, and the cells eluted and plated onto gelatin-coated coverslips in 6-well plates.

Heart suborgan fractionation. Cardiac cells were dissociated as described previously using Liberase $\mathrm{TH}(0.125 \mathrm{U} / \mathrm{ml})$ (MilliporeSigma) (56). The cell suspension was filtered through a $100-\mu \mathrm{m}$ strainer and centrifuged at $50 \mathrm{~g}$ for 5 minutes. CMs were pelleted and subjected to Percoll density gradient centrifugation (1,200 $g$ for $30 \mathrm{~min}$ ) for RNA and protein isolation. Non-CMs in the supernatant were centrifuged at $500 \mathrm{~g}(10 \mathrm{~min})$ and prepared for FACS. RBC in non-CMs were lysed and pellets resuspended in staining buffer (2\% FBS/PBS, $2 \mathrm{mM}$ EDTA, and $100 \mathrm{U} / \mathrm{ml}$ RNase inhibitor; catalog M0314L, New England BioLabs). Incubation with calcein $\mathrm{AM}(100 \mathrm{nM}$, room temperature, $30 \mathrm{~min}$ ) and then with CD45 and CD31 antibodies (catalogs 103114 and 102508 , BioLegend) on ice (30 min) was followed by 7-AAD ( 5 $\mu \mathrm{g} / \mathrm{ml}$ ) for an additional 10 minutes before the end of the incubation. Cells were pelleted, resuspended, filtered (70- $\mu \mathrm{m}$ Flowmi cell strainer), and sorted on a FACSAria Fusion (BD Biosciences). Singlet cells were gated by forward scatter area (FSC-A) and forward scatter height (FSC-H). Additional gating of live (calcein $\mathrm{AM}^{+} 7-\mathrm{AAD}^{-}$) cells was performed. CD $45^{-} \mathrm{CD} 31^{+} \mathrm{EC}$ fractions were collected for RNA isolation.

ISH. CD36 mRNA expression was detected in frozen sections via ISH using probes and the QuantiGene ViewRNA tissue assay (Affymetrix) according to the manufacturer's instructions. No probe controls or tissues from $\mathrm{Cd} 36-\mathrm{KO}$ mice were used for negative controls.

LCM. Mouse heart tissues were filled with OCT compound. Polyethylene naphthalate (PEN) membrane glass slides were prepared for cryosectioning. The tissue block was cryosectioned entirely at $10 \mu \mathrm{m}$ and stained with H\&E for histomorphologic identification of cardiomyocytes. A Leica DM6000 B microscope was used for cardiomyocyte harvesting. Captured cardiomyocytes were subjected to RNA purification (ARCTURUS PicoPure RNA Isolation Kit, Applied Biosystems, Thermo Fisher Scientific) and cDNA amplification (The Ovation Pico WTA System V2, NuGEN).

Tissue gene expression. Four-month-old mice were fasted for sixteen hours. Total RNA was prepared using a Pure Link Micro-to-Midi Total Purification Kit (Invitrogen, Thermo Fisher Scientific). RNA $(1 \mu \mathrm{g})$ was treated with DNase I (Invitrogen, Thermo Fisher Scientific) for 15 minutes and reverse transcribed using the ThermoScript RT-PCR Kit (Invitrogen, Thermo Fisher Scientific). An ABI 7700 system (Applied Biosystems, Thermo Fisher Scientific) and SYBR Green PCR Master Mix (Applied Biosystems, Thermo Fisher Scientific) were used for qRT-PCR. For analysis, we used Sequence Detection Software (Applied Biosystems, Thermo Fisher Scientific) and standard curves generated using undiluted and diluted (1:10, 1:100, and 1:1,000) cDNA heart tissue samples. Correlation coefficients were 0.98 or greater. Data were normalized to $18 \mathrm{~S}$ rRNA.

RNA-seq data analysis. Raw sequencing data were received in FASTQ format. Read mapping used Tophat 2.0.9 against the mm10 mouse reference genome. The resulting BAM alignment files were processed using the HTSeq 0.6.1 python framework and respective mm10 GTF gene annotation (UCSC database). The Bioconductor package DESeq2 (3.2) was used to identify differentially expressed genes (DEGs) and for statistical analysis based on a negative binomial distribution model. The resulting values were adjusted using the Benjamini-Hochberg method for FDR determination. Genes with an adjusted $P$ value of less than 0.05 were determined to be differentially expressed, and KEGG Analysis was performed to identify top canonical pathways being altered. RNA-seq data were deposited in the NCBI's Gene Expression Omnibus (GEO) database (GEO GSE116350).

Electron microscopy. Left ventricles from 16-hour-fasted 4-monthold male mice were fixed with $2.5 \%$ glutaraldehyde and $2 \%$ paraformaldehyde in 0.1M sodium cacodylate buffer ( $\mathrm{pH}$ 7.2) for 2 hours and then postfixed with $1 \%$ osmium tetroxide ( $1.5 \mathrm{~h}$ at room temperature), processed, and embedded in EMbed 812 (Electron Microscopy Sciences). Ultrathin sections (60-nm) were cut (Leica UC6 microtome), mounted onto 200 mesh copper grids, and stained with uranyl acetate and lead citrate. Stained grids were examined with a Philips CM-12 electron microscope and photographed with a Gatan $(4 \mathrm{k} \times 2.7 \mathrm{k})$ digital camera. Lipid droplets were counted in random $100-\mu \mathrm{m}^{2}$ areas.

Uptake of $\left[{ }^{3} \mathrm{H}\right]$ oleic acid and $\left[{ }^{14} \mathrm{C}\right]$ hexanoic acid. Cd36 $6^{f / f l}, \mathrm{EC}-\mathrm{Cd} 36^{-/}$, and $\mathrm{CM}-\mathrm{Cd} 36^{-/-}$mice (4-month-old, 16-hour-fasted) were injected i.v. 
with $100 \mu \mathrm{l}$ PBS containing $1 \times 10^{6} \mathrm{dpm}$ of $\left[{ }^{3} \mathrm{H}\right]$ oleic acid and $1 \times 10^{6}$ of $\left[{ }^{14} \mathrm{C}\right]$ hexanoic acid (PerkinElmer). Mouse plasma volume was estimated as $0.55 \mathrm{ml} / \mathrm{kg} \mathrm{BW}$ (57). Plasma radioactive concentration was calculated by BW $(\mathrm{mg}) \times(0.55 \mathrm{ml} / 1,000 \mathrm{mg}) \times 10^{6}(\mathrm{dpm})$. Blood was collected $0,30,120$, and 300 seconds after injection for radioactive counts (10 $\mu \mathrm{l}$ plasma). The heart vasculature was perfused with 10 $\mathrm{ml}$ PBS via cardiac puncture 5 minutes after injection. Tissues were excised and accumulated radioactivity measured. Tissue oleic and hexanoic acid uptake was adjusted by plasma radioactivity at $30 \mathrm{~min}$ utes and normalized to that of littermate controls.

PET studies. Differences in FA metabolism and FDG were evaluated with PET imaging of 4- to 5-month-old 16-hour-fasted mice using $\left[{ }^{11} \mathrm{C}\right]$ palmitate and $\left[{ }^{18} \mathrm{~F}\right] \mathrm{DG}$ as described previously (58). The uptake and transport of tracer were visualized and quantified by summing the uptake kinetics up to 60 minutes after tracer administration. All images and corresponding data were normalized to the dose and weight of the animal and presented as the standardized uptake value (SUV = activity $\times$ weight/injected dose).

Statistics. Results are expressed as the mean \pm SD. Significant differences between groups were determined by an ANOVA $t$ test or a 1-way ANOVA multiple comparisons test. The AUC value was evaluated using the trapezoid method. A $P$ value of less than 0.05 was considered significant.

Study approval. The IRBs of Columbia University and New York University Medical Center approved all study protocols. Generation of the mice and all metabolic and genetic studies were reviewed and approved by the IACUCs of New York University and Washington University.

\section{Author contributions}

NHS, NAA, and IJG designed the study and wrote the manuscript. NHS created the targeting constructs for the genetically modified mice and performed experiments assessing gene expression, plasma metabolites, and tissue analysis. TAP assisted with construct design and validation of the mouse models. FW, XF, and SQY performed the radioactive FA uptake experiments. HRC created the LysM-Cre $C d 36^{-/-}$mice and obtained data on their circulating lipid and glucose levels. D. Basu, D. Scerbo, TAP, STY, VSP, FS, SB, and LAH performed experiments. KIS performed and analyzed the PET studies. D. Scerbo and TL contributed to data analysis and discussion. KD, AEM, and KIS performed ISH and heart cell fractionation studies. NG edited the manuscript. KK assisted with Western blotting for insulin signaling analysis.

\section{Acknowledgments}

We thank Stephanie Chiang and Sunny Son for help with manuscript preparation and data analysis, Yun-ying Hu (New York University School of Medicine) for the kinetic studies, and Chyuan-Sheng Lin at the Columbia University Transgenic mouse Core for the generation of floxed mice by pronuclear DNA microinjection and embryo transfer. We thank the New York University Langone Medical Center OCS Microscopy Core, specifically Alice Liang, Kristen Dancel, and Yan Deng, for their consultation and assistance with transmission electron microscopic work. We also thank Nikki Fettig at the Radiological Chemistry and Imaging Laboratory (RCIL) of the Mallinckrodt Institute of Radiology at Washington University for assistance with the $\left[{ }^{11} \mathrm{C}\right]$ palmitate and $\left[{ }^{18} \mathrm{~F}\right] D G$ studies. We also thank A. Tall (Columbia University Medical Center), C. Semenkovich (Washington University School of Medicine), R. Kitsis (Department of Medicine, Albert Einstein College of Medicine, New York, New York, USA), and W. Blaner (Columbia University Medical Center) for constructive comments made during the preparation of this manuscript. The studies were funded by National Heart, Blood, and Lung Institute grants HL45095 and HL73029 (to IJG) and DK33301 and DK11175 (to NAA).

Address correspondence to: Ira J. Goldberg, Division of Endocrinology, Diabetes and Metabolism, NYU Langone Medical Center, Science Building 617, 435 E. 30th Street, New York, New York 10016, USA. Phone: 646.501.0589; Email: Ira.Goldberg@nyumc. org. Or to: Nada A. Abumrad, Department of Medicine, Washington University School of Medicine, 660 S Euclid Avenue, Campus Box 8031, St. Louis, Missouri 63110, USA. Phone: 314.747.0348; Email: Nabumrad@wustl.edu.
1. Stern JH, Rutkowski JM, Scherer PE. Adiponectin, leptin, and fatty acids in the maintenance of metabolic homeostasis through adipose tissue crosstalk. Cell Metab. 2016;23(5):770-784.

2. Sorrentino D, Robinson RB, Kiang CL, Berk PD. At physiologic albumin/oleate concentrations oleate uptake by isolated hepatocytes, cardiac myocytes, and adipocytes is a saturable function of the unbound oleate concentration. Uptake kinetics are consistent with the conventional theory. J Clin Invest. 1989;84(4):1325-1333.

3. Murota K, Storch J. Uptake of micellar longchain fatty acid and sn-2-monoacylglycerol into human intestinal Caco- 2 cells exhibits characteristics of protein-mediated transport. J Nutr. 2005;135(7):1626-1630.

4. Abumrad NA, Goldberg IJ. CD36 actions in the heart: Lipids, calcium, inflammation, repair and more? Biochim Biophys Acta. 2016;1861(10):1442-1449.

5. Silverstein RL, Febbraio M. CD36, a scaven- ger receptor involved in immunity, metabolism, angiogenesis, and behavior. Sci Signal. 2009;2(72):re3.

6. Endemann G, Stanton LW, Madden KS, Bryant CM, White RT, Protter AA. CD36 is a receptor for oxidized low density lipoprotein. J Biol Chem. 1993;268(16):11811-11816.

7. Abumrad NA, el-Maghrabi MR, Amri EZ, Lopez E, Grimaldi PA. Cloning of a rat adipocyte membrane protein implicated in binding or transport of longchain fatty acids that is induced during preadipocyte differentiation. Homology with human CD36. J Biol Chem. 1993;268(24):17665-17668.

8. Hsieh FL, Turner L, Bolla JR, Robinson CV, Lavstsen T, Higgins MK. The structural basis for CD36 binding by the malaria parasite. Nat Commun. 2016;7:12837.

9. Nahlé Z, et al. CD36-dependent regulation of muscle FoxO1 and PDK4 in the PPAR delta/ beta-mediated adaptation to metabolic stress. J Biol Chem. 2008;283(21):14317-14326.

10. Samovski D, et al. Regulation of AMPK activation by CD36 links fatty acid uptake to $\beta$-oxidation. Diabetes. 2015;64(2):353-359.

11. Coburn CT, Knapp FF, Febbraio M, Beets AL, Silverstein RL, Abumrad NA. Defective uptake and utilization of long chain fatty acids in muscle and adipose tissues of CD36 knockout mice. JBiol Chem. 2000;275(42):32523-32529.

12. Febbraio M, et al. A null mutation in murine CD36 reveals an important role in fatty acid and lipoprotein metabolism. J Biol Chem. 1999;274(27):19055-19062.

13. Drover VA, et al. CD36 deficiency impairs intestinal lipid secretion and clearance of chylomicrons from the blood. JClin Invest. 2005;115(5):1290-1297.

14. Nozaki S, et al. CD36 mediates long-chain fatty acid transport in human myocardium: complete myocardial accumulation defect of radiolabeled longchain fatty acid analog in subjects with CD36 deficiency. Mol Cell Biochem. 1999;192(1-2):129-135.

15. Ma X, et al. A common haplotype at the CD36 locus is associated with high free fatty acid levels and increased cardiovascular risk in Caucasians. 
Hum Mol Genet. 2004;13(19):2197-2205.

16. Wang Y, et al. Ephrin-B2 controls VEGF-induced angiogenesis and lymphangiogenesis. Nature. 2010;465(7297):483-486.

17. Hevener A, Reichart D, Janez A, Olefsky J. Female rats do not exhibit free fatty acid-induced insulin resistance. Diabetes. 2002;51(6):1907-1912.

18. Priego T, Sánchez J, Picó C, Palou A. Sex-differential expression of metabolism-related genes in response to a high-fat diet. Obesity (Silver Spring). 2008;16(4):819-826.

19. Masuda D, et al. Chylomicron remnants are increased in the postprandial state in CD36 deficiency. JLipid Res. 2009;50(5):999-1011.

20. Hajri T, Han XX, Bonen A, Abumrad NA. Defective fatty acid uptake modulates insulin responsiveness and metabolic responses to diet in CD36-null mice. JClin Invest. 2002;109(10):1381-1389.

21. Lundsgaard AM, Kiens B. Gender differences in skeletal muscle substrate metabolism - molecular mechanisms and insulin sensitivity. Front Endocrinol (Lausanne). 2014;5:195.

22. Fukuchi K, et al. Enhanced myocardial glucose use in patients with a deficiency in long-chain fatty acid transport (CD36 deficiency). J Nucl Med.1999;40(2):239-243.

23. Trent CM, et al. Lipoprotein lipase activity is required for cardiac lipid droplet production. JLipid Res. 2014;55(4):645-658.

24. Liu L, et al. Paradoxical coupling of triglyceride synthesis and fatty acid oxidation in skeletal muscle overexpressing DGAT1. Diabetes. 2009;58(11):2516-2524.

25. Monetti M, et al. Dissociation of hepatic steatosis and insulin resistance in mice overexpressing DGAT in the liver. Cell Metab. 2007;6(1):69-78.

26. Ibrahimi A, et al. Muscle-specific overexpression of FAT/CD36 enhances fatty acid oxidation by contracting muscle, reduces plasma triglycerides and fatty acids, and increases plasma glucose and insulin. J Biol Chem. 1999;274(38):26761-26766.

27. Nickerson JG, et al. Greater transport efficiencies of the membrane fatty acid transporters FAT/ CD36 and FATP4 compared with FABPpm and FATP1 and differential effects on fatty acid esterification and oxidation in rat skeletal muscle. JBiol Chem. 2009;284(24):16522-16530.

28. Coort SL, et al. Sulfo-N-succinimidyl esters of long chain fatty acids specifically inhibit fatty acid translocase (FAT/CD36)-mediated cellular fatty acid uptake. Mol Cell Biochem. 2002;239(1-2):213-219.

29. Jay AG, Chen AN, Paz MA, Hung JP, Hamilton JA. CD36 binds oxidized low density lipoprotein (LDL) in a mechanism dependent upon fatty acid binding. J Biol Chem. 2015;290(8):4590-4603.

30. Bartelt A, et al. Brown adipose tissue activity controls triglyceride clearance. Nat Med. 2011;17(2):200-205.

31. Kanda T, et al. PPARgamma in the endothelium regulates metabolic responses to high-fat diet in mice. J Clin Invest. 2009;119(1):110-124.

32. Coppiello G, et al. Meox2/Tcf15 heterodimers program the heart capillary endothelium for cardiac fatty acid uptake. Circulation. 2015;131(9):815-826.

33. Hagberg CE, et al. Targeting VEGF-B as a novel treatment for insulin resistance and type 2 diabetes. Nature. 2012;490(7420):426-430.

34. Kivelä R, et al. VEGF-B-induced vascular growth leads to metabolic reprogramming and ischemia resistance in the heart. $E M B O \mathrm{Mol}$ Med 2014;6(3):307-321.

35. Jang C, et al. A branched-chain amino acid metabolite drives vascular fatty acid transport and causes insulin resistance. Nat Med. 2016;22(4):421-426.

36. Salameh A, et al. Prohibitin/annexin 2 interaction regulates fatty acid transport in adipose tissue. JCI Insight. 2016;1(10):e86351.

37. Nolan DJ, et al. Molecular signatures of tissue-specific microvascular endothelial cell heterogeneity in organ maintenance and regeneration. Dev Cell. 2013;26(2):204-219.

38. Glatz JF, Nabben M, Heather LC, Bonen A, Luiken JJ. Regulation of the subcellular trafficking of CD36, a major determinant of cardiac fatty acid utilization. Biochim Biophys Acta. 2016;1861(10):1461-1471.

39. Angin Y, et al. CD36 inhibition prevents lipid accumulation and contractile dysfunction in rat cardiomyocytes. Biochem J. 2012;448(1):43-53.

40. Sung MM, et al. Cardiomyocyte-specific ablation of CD36 accelerates the progression from compensated cardiac hypertrophy to heart failure. Am J Physiol Heart Circ Physiol. 2017;312(3):H552-H56O.

41. Guerre-Millo M, et al. PPAR-alpha-null mice are protected from high-fat diet-induced insulin resistance. Diabetes. 2001;50(12):2809-2814.

42. Kotani K, Peroni OD, Minokoshi Y, Boss O, Kahn BB. GLUT4 glucose transporter deficiency increases hepatic lipid production and peripheral lipid utilization. JClin Invest. 2004;114(11):1666-1675.

43. Tordjman K, et al. PPARalpha deficiency reduces insulin resistance and atherosclerosis in apoEnull mice. JClin Invest. 2001;107(8):1025-1034.

44. Augustus A, et al. Cardiac-specific knock-out of lipoprotein lipase alters plasma lipoprotein triglyceride metabolism and cardiac gene expres- sion. J Biol Chem. 2004;279(24):25050-25057.

45. Samovski D, et al. Regulation of insulin receptor pathway and glucose metabolism by CD36 signaling. Diabetes. 2018;67(7):1272-1284.

46. Leprêtre F, et al. Genetic study of the CD36 gene in a French diabetic population. Diabetes Metab. 2004;30(5):459-463.

47. Miyaoka K, Kuwasako T, Hirano K, Nozaki S, Yamashita S, Matsuzawa Y. CD36 deficiency associated with insulin resistance. Lancet. 2001;357(9257):686-687.

48. Kuang M, Febbraio M, Wagg C, Lopaschuk GD, Dyck JR. Fatty acid translocase/CD36 deficiency does not energetically or functionally compromise hearts before or after ischemia. Circulation 2004;109(12):1550-1557.

49. Nagendran J, et al. Cardiomyocyte-specific ablation of CD36 improves post-ischemic functional recovery. J Mol Cell Cardiol. 2013;63:180-188.

50. Irie $\mathrm{H}$, et al. Myocardial recovery from ischemia is impaired in CD36-null mice and restored by myocyte $\mathrm{CD} 36$ expression or medium-chain fatty acids. Proc Natl Acad Sci USA. 2003;100(11):6819-6824.

51. Goldberg IJ, Trent CM, Schulze PC. Lipid metabolism and toxicity in the heart. Cell Metab. 2012;15(6):805-812.

52. Lopaschuk GD, Ussher JR, Folmes CD, Jaswal JS, Stanley WC. Myocardial fatty acid metabolism in health and disease. Physiol Rev. 2010;90(1):207-258.

53. Howard MD, Hood ED, Greineder CF, Alferiev IS, Chorny M, Muzykantov V. Targeting to endothelial cells augments the protective effect of novel dual bioactive antioxidant/anti-inflammatory nanoparticles. Mol Pharm. 2014;11(7):2262-2270.

54. Folch J, Lees M, Sloane Stanley GH. A simple method for the isolation and purification of total lipides from animal tissues. J Biol Chem. 1957;226(1):497-509.

55. Seo T, et al. Lipoprotein lipase-mediated selective uptake from low density lipoprotein requires cell surface proteoglycans and is independent of scavenger receptor class B type 1. J Biol Chem. 2000;275(39):30355-30362.

56. Louch WE, Sheehan KA, Wolska BM. Methods in cardiomyocyte isolation, culture, and gene transfer. J Mol Cell Cardiol. 2011;51(3):288-298.

57. Riches AC, Sharp JG, Thomas DB, Smith SV. Blood volume determination in the mouse. JPhysiol (Lond). 1973;228(2):279-284.

58. Burkart EM, et al. Nuclear receptors PPARbeta/ delta and PPARalpha direct distinct metabolic regulatory programs in the mouse heart. J Clin Invest. 2007;117(12):3930-3939. 